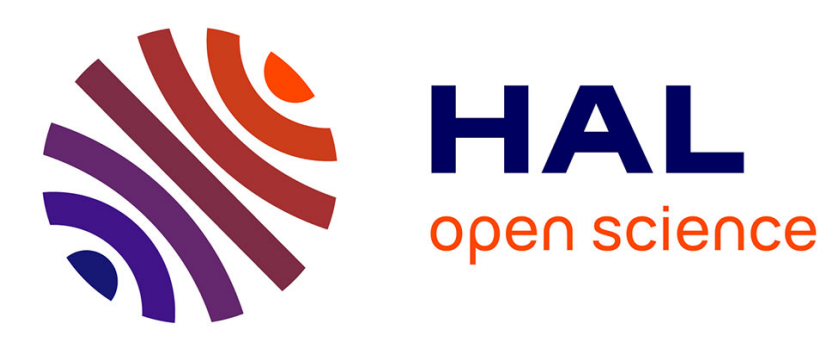

\title{
Nonlinear vibrations of a mechanical system with non-regular nonlinearities and uncertainties
}

\author{
Jérôme Didier, Jean-Jacques Sinou, Beatrice Faverjon
}

\section{To cite this version:}

Jérôme Didier, Jean-Jacques Sinou, Beatrice Faverjon. Nonlinear vibrations of a mechanical system with non-regular nonlinearities and uncertainties. Communications in Nonlinear Science and Numerical Simulation, 2013, 18 (11), pp.3250-3270. 10.1016/j.cnsns.2013.03.005 . hal-00831862

\section{HAL Id: hal-00831862 \\ https://hal.science/hal-00831862}

Submitted on 7 Jun 2013

HAL is a multi-disciplinary open access archive for the deposit and dissemination of scientific research documents, whether they are published or not. The documents may come from teaching and research institutions in France or abroad, or from public or private research centers.
L'archive ouverte pluridisciplinaire HAL, est destinée au dépôt et à la diffusion de documents scientifiques de niveau recherche, publiés ou non, émanant des établissements d'enseignement et de recherche français ou étrangers, des laboratoires publics ou privés. 


\title{
Nonlinear vibrations of a mechanical system with non-regular nonlinearities and uncertainties
}

\author{
J. $\operatorname{Didier}^{a}$, J-J. Sinou ${ }^{a}$, B. Faverjon ${ }^{b}$ \\ ${ }^{a}$ Laboratoire de Tribologie et Dynamique des Systèmes UMR 5513, Ecole Centrale de Lyon, France. \\ ${ }^{b}$ Laboratoire de Mécanique des Contacts et des Structures UMR 5259, INSA Lyon, France.
}

\begin{abstract}
This paper presents the concept of the Stochastic Multi-dimensional Harmonic Balance Method (Stochastic-MHBM) in order to solve dynamical problems with non-regular nonlinearities in presence of uncertainties. To treat the nonlinearity in the stochastic and frequency domains, the Alternate Frequency-Time method with Probabilistic Collocation (AFTPC) is proposed. The approach is demonstrated using nonlinear twodegree-of-freedom model with different types of nonlinearities (cubic nonlinearity, contact/no contact, friction). The quasi-periodic stochastic dynamic response is evaluated considering uncertainties in linear and nonlinear parts of the mechanical system. The results are compared with those obtained from the classical Monte Carlo Simulation (MCS). For various numerical tests, it is found that the results agreed very well whilst requiring significantly less computation.
\end{abstract}

\section{Introduction}

Due to the fact that deterioration or evolution of the structure during its lifetime can drastically affect its vibration behavior, there has been a crucial and strong development in the treatment of variability in mechanical system and in the application of methods to enable the analysis of stochastic vibration problems. In order to estimate the stochastic dynamic response of a linear system, several approaches can be used. One classical method consists in carrying out Monte Carlo simulations (MCS) to obtain for instance the probability density function or the envelope of the random dynamic response. This non-intrusive in a stochastic sense method can easily be done, however, the convergence of the method needs a high number of samples that means a high CPU time. One possibility to reduce this CPU time is to associate one non-intrusive method called the Probabilistic Collocation (PC) [1] method and consisting in calculating the deterministic problem on the collocation points, points Gaussian quadrature, alone, of number smaller than that for the classical MC approach. Besides, the Monte Carlo simulations, the perturbation methods are based on the development of the random quantities in Taylor series [2] or Neumann series [3, 4]. These methods give good results for random problems with small variations but do not seem suitable to dynamic problems for frequencies close to the resonance. Moreover, estimating the envelope of the response using the intervals method can be possible, however this technique tends to over-estimate the results ; then it can not be adapted to calculate the random dynamic response with reliability. Another method that could be suitable for convenient random variation is the Polynomial Chaos Expansion (PCE) [5]. The Polynomial Chaos Expansion has already been successfully applied to estimate evolutions of the periodic and quasi-periodic responses with harmonic components of a rotor system with uncertainties [6, 7].

Furthermore, the need for consideration of nonlinear effects in the description of a dynamical system is well recognized in the field of engineering and numerous studies have been conducted to understand and model the nonlinear phenomena in structural dynamics during the past decades [8]. Even if most of these models deal with deterministic parameters, it is obvious that variations in the geometry or material properties are often present in these systems as it has already been mentioned in the previous paragraph for linear systems. However, the treatment of uncertainty in the nonlinear structures is not common. So the effects of variations in the geometry or material properties on the nonlinear dynamic responses remain misunderstood. Moreover, it can be noted that most of the classical stochastic methods used in the linear case fail to deal with a nonlinear problem. For example, the previous methodology proposed by the authors in $[6,7]$ as well as the probabilistic collocation method do not allow to solve problems including returning points [9] since they are unable to describe multi-solutions as usually observed for nonlinear systems.

In the present study, we propose a new formulation, called the Stochastic Multi-dimensional Harmonic Balance Method (Stochastic-MHBM) to solve the stochastic nonlinear dynamic problems. The aim of this new numerical procedure is to evaluate the nonlinear responses of mechanical systems with uncertainties. The nonlinear system can include both regular and nonregular nonlinearities and it can be submitted to mono or multi-frequency excitations. Uncertainties will relate just as easily to nonlinearities that on the linear terms (rigidity, damping and mass).

The next section of this paper presents the Stochastic Multi-dimensional Harmonic Balance Method in the order to determine the quasi-periodic stochastic nonlinear response. Also, a new methodology called the Alternate Frequency Time method with 
Probabilistic Collocation (AFTPC) that is based on the combination of the Alternate Frequency-Time with Probabilistic Collocation will be developed. This theoretical development (i.e. Alternate Frequency-Time method with Probabilistic Collocation, AFTPC) allows the determination of the nonlinear terms in the stochastic and frequency domains. In the third section, the modeling of the problem as well as the types of the nonlinearities treated will be explained. Effects of the following three kinds of nonlinearities will be examined: cubic stiffness, contact/no contact, systems with frictional interface. Finally, results will be presented for several values of the random parameters, then will be compared to results obtained from the Monte Carlo simulations with the Multi-dimensional Harmonic Balance Method. To demonstrate the robustness and efficiency of the proposed methodology, the nonlinear dynamic response of mechanical system with various uncertainties will be investigated for mono or multiple excitation frequencies. The mean and standard deviation outputs will not be used since they do not allow to represent multi-solutions.

\section{Stochastic Multi-dimensional Harmonic Balance Method}

In this section, we propose to describe the Stochastic Multi-dimensional Harmonic Balance Method based on the extension and coupling procedure of the Multi-dimensional Harmonic Balance Method with the Polynomial Chaos Expansion. We recall that the objective is to propose a new methodology to obtain the random quasi-periodic dynamic response of a nonlinear system (with regular or non-regular nonlinearities) subjected to multi-frequency excitations in the presence of uncertainty on several physical parameters.

First of all, a brief review of the Multi-dimensional Harmonic Balance Method (MHBM) is presented in this section. The MHBM approach will be used in the following numerical studies in order to estimate the nonlinear responses via the Monte Carlo simulations. We recall that the Monte Carlo approach will serve as reference to demonstrate the effectiveness and validity of the proposed Stochastic Multi-dimensional Harmonic Balance Method. Then, the second part of this section concerns the presentation of the proposed Stochastic Multi-dimensional Harmonic Balance Method.

\subsection{Multi-dimensional Harmonic Balance Method}

\subsubsection{General theory of the Multi-Harmonic Balance Method}

The general formulation for a nonlinear dynamical system can be written in the following form

$$
\mathbf{M} \ddot{\mathbf{x}}(t)+\mathbf{C} \dot{\mathbf{x}}(t)+\mathbf{K x}(t)=\mathbf{g}(t, \mathbf{x}(t))=\mathbf{f}(t)+\mathbf{f}_{\mathbf{n l}}(\mathbf{x}(t))
$$

where $\mathbf{M}, \mathbf{C}$ and $\mathbf{K}$ define respectively the mass, damping and stiffness matrices. $\mathbf{f}$ et $\mathbf{f}_{\mathbf{n l}}$ are the external forces and nonlinear effects in the system. The upper dot denotes a derivative with respect to time $t$.

Considering that the nonlinear system can be subjected to $p$ multiple periodic incommensurable excitation frequencies $\omega_{1}, \omega_{2}, \ldots, \omega_{p}$, the response of the system contains the frequency components of any linear combination of the incommensurable frequency components $k_{1} \omega_{1}+k_{2} \omega_{2}+\ldots+k_{i} \omega_{i}+\ldots+k_{p} \omega_{p}$ with $k_{i}=-N_{h},-N_{h}+1, \ldots,-1,0,1, \ldots, N_{h}-1, N_{h}$ where $N_{h}$ is the order of the Fourier series. Therefore the quasi-periodic dynamic response $\mathbf{x}(t)$ and the global excitation $\mathbf{g}(t, \mathbf{x}(t))$ including the external forces and nonlinear effects can be approximated in the form of multiple Fourier series [10]:

$$
\begin{aligned}
& \mathbf{x}(t)=\sum_{k_{1}=-N_{h}}^{N_{h}} \sum_{k_{2}=-N_{h}}^{N_{h}} \ldots \sum_{k_{p}=-N_{h}}^{N_{h}}\left(\mathbf{X}_{\left[k_{1}, k_{2}, \ldots, k_{p}\right]}^{\mathbf{c}_{1}} \cos \left(\sum_{i=1}^{p} k_{i} \omega_{i} t\right)+\mathbf{X}_{\left[k_{1}, k_{2}, \ldots, k_{p}\right]}^{\mathbf{s}_{3}} \sin \left(\sum_{i=1}^{p} k_{i} \omega_{i} t\right)\right) \\
& \mathbf{g}(t, \mathbf{x}(t))=\sum_{k_{1}=-N_{h}}^{N_{h}} \sum_{k_{2}=-N_{h}}^{N_{h}} \ldots \sum_{k_{p}=-N_{h}}^{N_{h}}\left(\mathbf{G}_{\left[k_{1}, k_{2}, \ldots, k_{p}\right]}^{\mathbf{c}} \cos \left(\sum_{i=1}^{p} k_{i} \omega_{i} t\right)+\mathbf{G}_{\left[k_{1}, k_{2}, \ldots, k_{p}\right]} \sin \left(\sum_{i=1}^{p} k_{i} \omega_{i} t\right)\right)
\end{aligned}
$$

where $\mathbf{X}_{\left[k_{1}, k_{2}, \ldots, k_{p}\right]}, \mathbf{X}_{\left[k_{1}, k_{2}, \ldots, k_{p}\right]}, \mathbf{G}_{\left[k_{1}, k_{2}, \ldots, k_{p}\right]}^{\mathbf{s}_{1}}$ and $\mathbf{G}_{\left[k_{1}, k_{2}, \ldots, k_{p}\right]}^{\mathbf{s}_{1}}$ define the Fourier coefficients of order $\left[k_{1}, k_{2}, \ldots, k_{p}\right]$ for the quasi-periodic response $\mathbf{x}(t)$ and the global excitation $\mathbf{g}(t, \mathbf{x}(t))$ respectively. A definition given by Kim and Choi [11] for retaining $N_{h}$ harmonics in a multiple Fourier series can be given in the following form $\sum_{i=1}^{p}\left|k_{i}\right| \leq N_{h}$. For the reader comprehension, it can be noted that all harmonics at negative combination frequencies can be replaced by harmonic terms at positive combination frequencies due to the trigonometric relation [8]. So it may be concluded that only terms at positive combination frequencies can be retained in the nonlinear response and nonlinear force expression. So, the previous expression (2) can be rewritten in a condensed form:

$$
\mathbf{x}(t)=\sum_{\mathbf{k} \in \mathbb{Z}^{m}}\left(\mathbf{X}_{\mathbf{k}}^{\mathbf{c}} \cos (\mathbf{k} \cdot \boldsymbol{\omega} t)+\mathbf{X}_{\mathbf{k}}^{\mathbf{s}} \sin (\mathbf{k} \cdot \boldsymbol{\omega} t)\right)
$$

where the (.) denotes the dot product, $\mathbf{k}$ is the harmonic number vector of each frequency direction, $m$ defines the dimension of the final multi-dimensional basis where $\mathbf{k}_{\mathbf{j}}$ (with $j=0, \ldots, m$ ) represents the $j^{\text {th }}$ harmonic terms and $\boldsymbol{\omega}$ is the vector of $m$ incommensurable frequencies considered in the solution of the nonlinear system. So, $\mathbf{X}_{\mathbf{k}}^{\mathbf{c}}$ and $\mathbf{X}_{\mathbf{k}}^{\mathbf{s}}$ define the unknown Fourier 
coefficients of any linear combinations of the incommensurable frequency components for the quasi-periodic response of the nonlinear system.

Then, it can be observed that the global excitation $\mathbf{g}(t, \mathbf{x}(t))$ can also be expressed in the following form

$$
\mathbf{g}(t, \mathbf{x}(t))=\sum_{\mathbf{k} \in \mathbb{Z}^{m}}\left(\mathbf{G}_{\mathbf{k}}^{\mathbf{c}} \cos (\mathbf{k} \cdot \boldsymbol{\omega} t)+\mathbf{G}_{\mathbf{k}}^{\mathbf{s}} \sin (\mathbf{k} \cdot \boldsymbol{\omega} t)\right)
$$

For convenience, it is wise to deal with a non-dimensional time parameter $\mathbf{T}$ of dimension $p$. It refers to the hyper-time concept $\mathbf{T}=\left[T_{1}, \ldots, T_{p}\right]=\boldsymbol{\omega} t[12]$. Considering this last relation, Equation (4) can be rewritten by considering an equivalent function $\hat{\mathbf{x}}(t)$ of $p$ time variables in a $p$-dimensional time domain:

$$
\mathbf{x}(t)=\hat{\mathbf{x}}(\mathbf{T})=\hat{\mathbf{x}}(\boldsymbol{\omega} \mathbf{t})
$$

This last equation is $2 \pi$ periodic on every hyper-time dimension of T. Substituting expressions (4) and (5) in the equation of motion of the system (1), it can be rewritten in the form of a linear algebraic matrix equation system :

$$
\mathbf{L X}=\mathbf{F}+\mathbf{F}_{\mathbf{n l}}(\mathbf{X})
$$

where $\mathbf{L}$ is defined by $\mathbf{L}=\operatorname{diag}\left(\mathbf{K}, \mathbf{L}_{1}, \ldots, \mathbf{L}_{m}\right)$ with

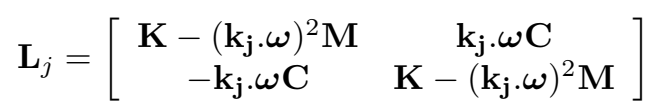

Then $\mathbf{X}, \mathbf{F}$ and $\mathbf{F}_{\mathbf{n l}}$ denote the unknown vector of harmonic coefficients, the projection of the external forces and the nonlinear part, respectively. They are given by respectively

$$
\begin{aligned}
& \mathbf{X}=\left[\begin{array}{llllll}
\mathbf{X}_{\mathbf{k}_{\mathbf{0}}}^{\mathbf{c} T} & \mathbf{X}_{\mathbf{k}_{\mathbf{1}}}^{\mathbf{c}} & \mathbf{X}_{\mathbf{k}_{\mathbf{1}}}^{\mathbf{s}} & \ldots & \mathbf{X}_{\mathbf{k}_{\mathbf{m}}}^{\mathbf{c}} & \mathbf{X}_{\mathbf{k}_{\mathbf{m}}}^{\mathbf{s}}
\end{array}\right]^{T} \\
& \mathbf{F}=\left[\begin{array}{llllll}
\mathbf{F}_{\mathbf{k}_{\mathbf{0}}}^{\mathbf{c} T} & \mathbf{F}_{\mathbf{k}_{\mathbf{1}}}^{\mathbf{c} T} & \mathbf{F}_{\mathbf{k}_{\mathbf{1}}}^{\mathbf{s} T} & \ldots & \mathbf{F}_{\mathbf{k}_{\mathbf{m}}}^{\mathbf{c} T} & \mathbf{F}_{\mathbf{k}_{\mathbf{m}}}^{\mathbf{s} T}
\end{array}\right]^{T}
\end{aligned}
$$

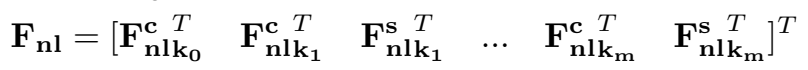

It may be difficult to determine the Fourier coefficients of the nonlinear terms $\mathbf{F}_{\mathbf{n l}}$ directly due to the complexity of the nonlinearities. In computational applications, the idea of the AFT method is to use Discrete Fourier Transformation (DFT) to derive the Fourier components of the nonlinear forces for given displacements in the frequency domain. So the generalization of the Alternating Frequency Time process $[13,14]$ to a p-dimensional frequency domain with a p-dimensional DFT is performed to calculate the Fourier coefficients of the nonlinear terms $\mathbf{F}_{\mathbf{n l}}$. The following diagram illustrates this procedure:

$$
\mathbf{X} \stackrel{\mathrm{IDFT}}{\longrightarrow} \mathbf{x}(t) \quad \longrightarrow \mathbf{F}_{\mathbf{n l}} \stackrel{\mathrm{DFT}}{\longrightarrow} \mathbf{f}_{\mathbf{n l}}(t)
$$

By rewriting the vector of the $m$ incommensurable frequencies $\boldsymbol{\omega}$ in the following form $\boldsymbol{\omega}=\omega_{1}\left[1, \alpha_{2}, \ldots, \alpha_{p}\right]=\omega_{1} \boldsymbol{\alpha}$ where $\alpha_{i}$ are irrational numbers, the linear algebraic matrix equation system (7) is given by

$$
\left(\mathbf{I 0}+\omega_{1} \mathbf{I} \mathbf{1}+\omega_{1}^{2} \mathbf{I} \mathbf{2}\right) \mathbf{X}=\mathbf{F}+\mathbf{F}_{\mathbf{n l}}
$$

where I0, I1 et $\mathbf{I} 2$ are defined by

$$
\mathbf{I 0}=\operatorname{diag}\left(\mathbf{K}, \mathbf{I} \mathbf{0}_{1}, \ldots, \mathbf{I} \mathbf{0}_{m}\right) \quad ; \quad \mathbf{I} \mathbf{1}=\operatorname{diag}\left(\mathbf{0}, \mathbf{I} \mathbf{1}_{1}, \ldots, \mathbf{I} \mathbf{1}_{m}\right) \quad ; \quad \mathbf{I} \mathbf{2}=\operatorname{diag}\left(\mathbf{0}, \mathbf{I} \mathbf{2}_{1}, \ldots, \mathbf{I} \mathbf{2}_{m}\right)
$$

with

$$
\mathbf{I 0}_{j}=\left[\begin{array}{cc}
\mathbf{K} & \mathbf{0} \\
\mathbf{0} & \mathbf{K}
\end{array}\right] \quad ; \quad \mathbf{I} 1_{j}=\left[\begin{array}{cc}
\mathbf{0} & \mathbf{k}_{\mathbf{j}} \cdot \boldsymbol{\alpha} \mathbf{C} \\
-\mathbf{k}_{\mathbf{j}} \cdot \boldsymbol{\alpha} \mathbf{C} & \mathbf{0}
\end{array}\right] \quad ; \quad \mathbf{I 2}_{j}=\left[\begin{array}{cc}
-\left(\mathbf{k}_{\mathbf{j}} \cdot \boldsymbol{\alpha}\right)^{2} \mathbf{M} & \mathbf{0} \\
\mathbf{0} & -\left(\mathbf{k}_{\mathbf{j}} \cdot \boldsymbol{\alpha}\right)^{2} \mathbf{M}
\end{array}\right]
$$

\subsubsection{Path following: continuation}

In mechanical systems, it may be useful to track the evolution of the system behavior for different operational points of interest while all the other parameters are kept constant. In this case, it may be useful to apply predictor and corrector mechanisms in order to estimate the nonlinear response as one of its parameter varies (for example the evolution of the excitation frequency of the system).

The predictor-corrector method, called continuation algorithm, is an algorithm that proceeds in two steps. It includes an algorithm to find the predicted points for the next solution by using the predictor, and another algorithm to obtain solutions from these predicted points using the corrector. Various techniques and numerical procedures exist for these two steps. In this section, we propose to give an overview of the most classical methods for both the predictor and corrector methods [8]. We focus on 
the resolution of the nonlinear system given by Equation (7). This resolution corresponds to the minimization of a function $H$ defined by

$$
H\left(\mathbf{X}, \omega_{1}\right)=\mathbf{L X}-\mathbf{F}-\mathbf{F}_{\mathbf{n l}}(\mathbf{X})
$$

In the following part of the paper, the $n^{t h}$ converged point is defined by $y^{(n)}=\left(\mathbf{X}^{(n)}, \omega_{1}^{(n)}\right)$, the predicted point is denoted by $y^{(n+1,0)}=\left(\mathbf{X}^{(n+1,0)}, \omega_{1}^{(n+1,0)}\right)$, and the $j^{t h}$ corrected point is defined by $y^{(n+1, j)}=\left(\mathbf{X}^{(n+1, j)}, \omega_{1}^{(n+1, j)}\right)$.

The predictor-corrector method needs a prediction distance denoted $\Delta s$ that defines the distance between two consecutive points. Classically, the following approximation of the curvilinear abscissa can be proposed

$$
\Delta s^{(n+1)}=\sqrt{\left(\mathbf{X}^{(n+1)}-\mathbf{X}^{(n)}\right)^{T}\left(\mathbf{X}^{(n+1)}-\mathbf{X}^{(n)}\right)+\left(\omega_{1}^{(n+1)}-\omega_{1}^{(n)}\right)^{2}}
$$

The value of this distance $\Delta s$ must be adjusted automatically and optimized according to changes (more or less abrupt) of the nonlinear response curve. In regions of high curvature, it will be necessary to reduce the prediction distance to better estimate the next solution of the system. On the contrary, in the case of an solution that does not change significantly with changes in the control parameter, the prediction distance may be increased to accelerate the calculations while keeping a good estimate of the solution [8].

Firstly, the predictor is intended to find the predicted point for the next solution by calculating a rough approximation of the desired quantity. The predicted points are found on an extension of the vector (i.e. direction and distance) connecting the current solution and the previous solution. It may be noted that the quality of the predictor governs the number of corrector iterations required to obtain accurate solutions. The concept of the most classical predictors (tangent predictor, secant predictor and Lagrange polynomials predictor) are illustrated in Figure 1.

The secant method is defined by the recurrence relation

$$
y^{(n+1,0)}=y^{(n)}+\Delta s \frac{y^{(n)}-y^{(n-1)}}{\left\|y^{(n)}-y^{(n-1)}\right\|}
$$

As can be seen from the iteration process, the secant method requires two initial values, $y^{(n)}$ and $y^{(n-1)}$, which should ideally be chosen to lie close to the root. The secant method assumes that the function is approximately linear in the local region of interest and the algorithm may not converge for functions that are not sufficiently smooth.

The tangent method uses only one initial value $y^{(n)}$. However, the evaluation of derivatives has to be performed in order to calculate tangent vector $\vec{t}=\left(\overrightarrow{t_{\mathbf{X}}}, t_{\omega_{1}}\right)$ to the curve at the point $y^{(n)}$. The tangent method is defined by the relation

$$
y^{(n+1,0)}=y^{(n)}+\Delta s \vec{t}
$$

If we compare this process with the secant method, we see that the tangent method requires the evaluation of both the function and its derivative at every step, while the secant method only requires the evaluation of the function. Therefore, the secant method may occasionally be faster in practice. The predictor of Lagrange polynomials is a linear combination of Lagrange basis polynomials. This predictor uses Lagrange polynomials $P_{d}$ of degree $d$ to extrapolate the curve defined by the $d+1$ previous points. The estimation of the following point can be predicted by the relation

$$
P_{d}(s)=\sum_{k_{0}=0}\left(\prod_{\substack{k=0 \\ k \neq k_{0}}}^{d} \frac{s-s^{(i-k)}}{s^{\left(i-k_{0}\right)}-s^{(i-k)}}\right) y^{\left(i-k_{0}\right)}
$$

where $s^{(k)}$ denotes curvilinear abscissa of point $y^{(k)}$. The predicted point $y^{(n+1,0)}$ of curvilinear abscissa $\Delta s$ is calculated by the relation

$$
y^{(n+1,0)}=P_{d}(\Delta s)
$$

Secondly, we consider the corrector in the predictor/corrector method. The corrector step refines the initial approximation, using the predicted points obtained by the predictor as initial values. For the reader comprehension, the converged solution satisfies the relation $H\left(\mathbf{X}, \omega_{1}\right)=0$ instead of the predicted solution. So, the objective of this second step is to obtain the converged solution from the predicted point. The concept of the most classical correctors (arc length, pseudo arc length and Moore-Penrose) are illustrated in Figure 1.

The arc length method proposes to add an additional constraint between the corrected point $y^{(n+1, j)}$ and the previous converged point $y^{(n)}$. The distance condition is defined by the following relation

$$
\forall j \geq 1,\left\|\mathbf{X}^{(n+1, j)}-\mathbf{X}^{(n)}\right\|^{2}+\left|\omega_{1}^{(n+1, j)}-\omega_{1}^{(n)}\right|^{2}=\left(\Delta s^{(n+1)}\right)^{2}
$$




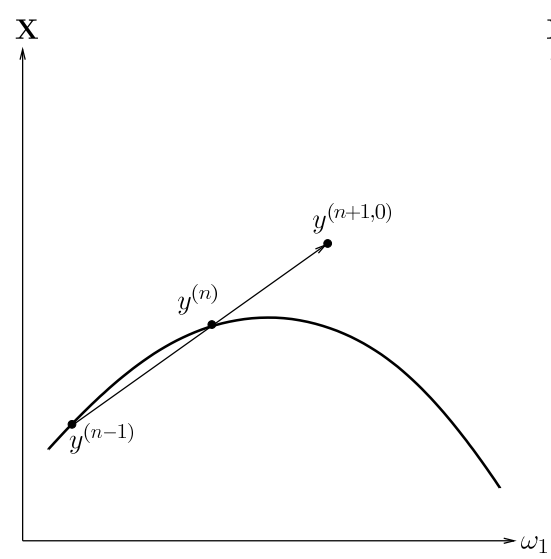

(a)

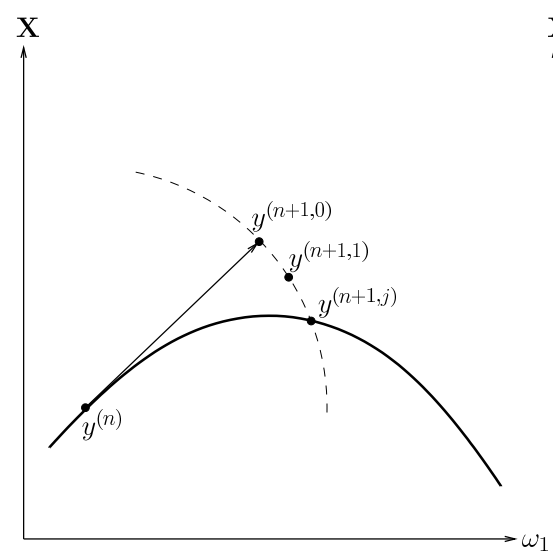

(d)

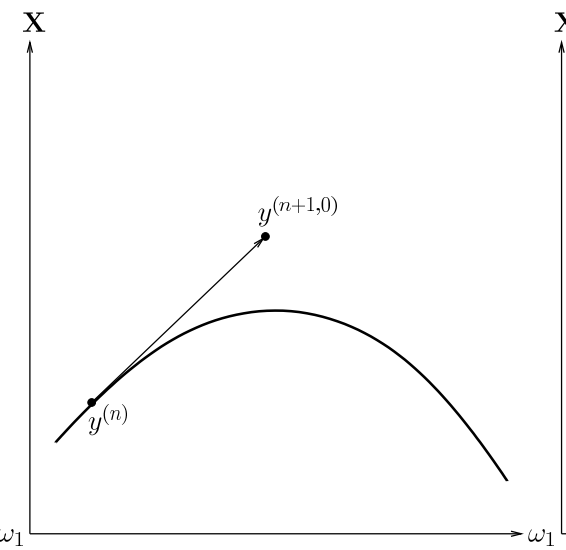

(b)

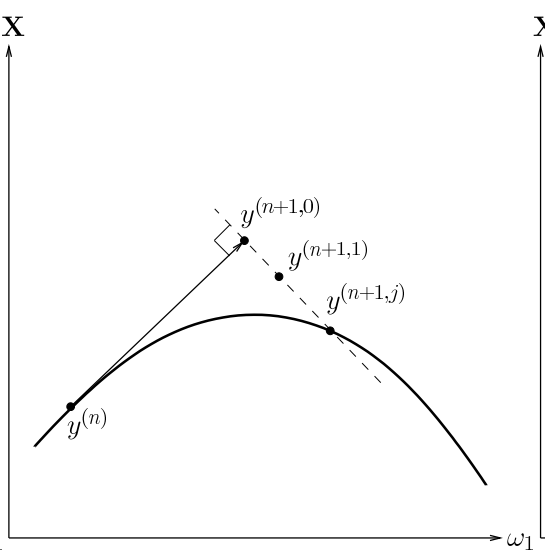

(e)

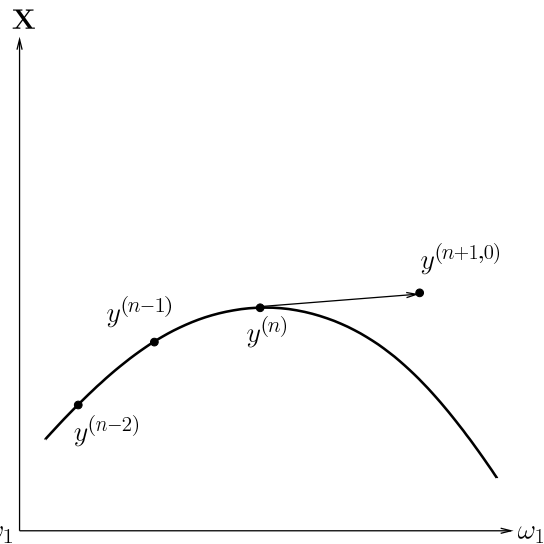

(c)

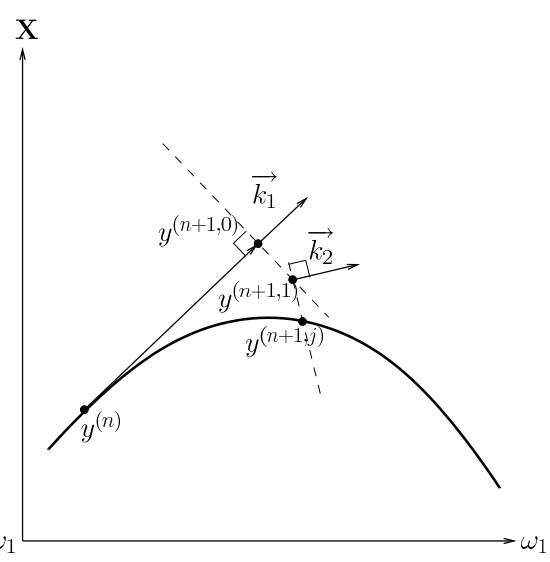

(f)

Fig. 1: Predictors and corrections (a) Secant method (b) Tangent method (c) Lagrange polynomials (d) Arc length (e) Pseudo arc length (f) Moore-Penrose.

The pseudo arc length method adds an orthogonality condition between the predicted point $y^{(n+1,0)}-y^{(n)}$ and the corrected points $\Delta y=y^{(n+1,0)}-y^{(n+1, j)}$. This constraint is given by

$$
\forall j \geq 1, \Delta y \cdot\left(y^{(n+1,0)}-y^{(n)}\right)=0
$$

After calculations, this constraint can be written in the following form

$$
\Delta \mathbf{X} \cdot\left(\mathbf{X}^{(n+1,0)}-\mathbf{X}^{(n)}\right)+\Delta \omega_{1} \cdot\left(\omega_{1}^{(n+1,0)}-\omega_{1}^{(n)}\right)=0
$$

where $\Delta \mathbf{X}$ and $\Delta \omega_{1}$ define the corrections for $\mathbf{X}$ and $\omega_{1}$. The Moore-Penrose method allows to solve the system by using the Moore-Penrose pseudo-inverse matrix of $\mathbf{A}$ defined by $\mathbf{A}^{+}=\mathbf{A}^{T}\left(\mathbf{A} \mathbf{A}^{T}\right)^{-1}$. The pseudo-inverse provides a least squares solution to a system of linear equations $\mathbf{A x}=\mathbf{b}$ where $\mathbf{A}$ has less rows than columns. Using this Moore-Penrose correctors, an additional orthogonality condition with the kernel of matrix $\mathbf{A}$ is implicitly added. This fact is illustrated in Figure $1(\mathrm{f})$ where $k_{j}$ defines the kernel for the $j^{t h}$ step of the corrector.

In the present study, the tangent and arc length methods will be used as predictor and corrector for the coupling of the Monte-Carlo simulations and Multi-Harmonic Balance Method.

\subsection{Stochastic Multi-dimensional Harmonic Balance Method}

Figure 2 represents the general algorithm procedure of the Stochastic Multi-dimensional Harmonic Balance Method that will be discussed and detailed in the next sections of the paper.

\subsubsection{The stochastic nonlinear problem}

Several excitations and materials parameters can be considered as random. Here, we retain for random quantities : mass, damping and rigidity parameters as well as the linear and nonlinear excitation terms respectively $\tilde{\mathbf{M}}(\tau), \tilde{\mathbf{C}}(\tau), \tilde{\mathbf{K}}(\tau), \tilde{\mathbf{F}}(\tau)$ and $\mathbf{F}_{\mathbf{n l}}(\tau)$, 


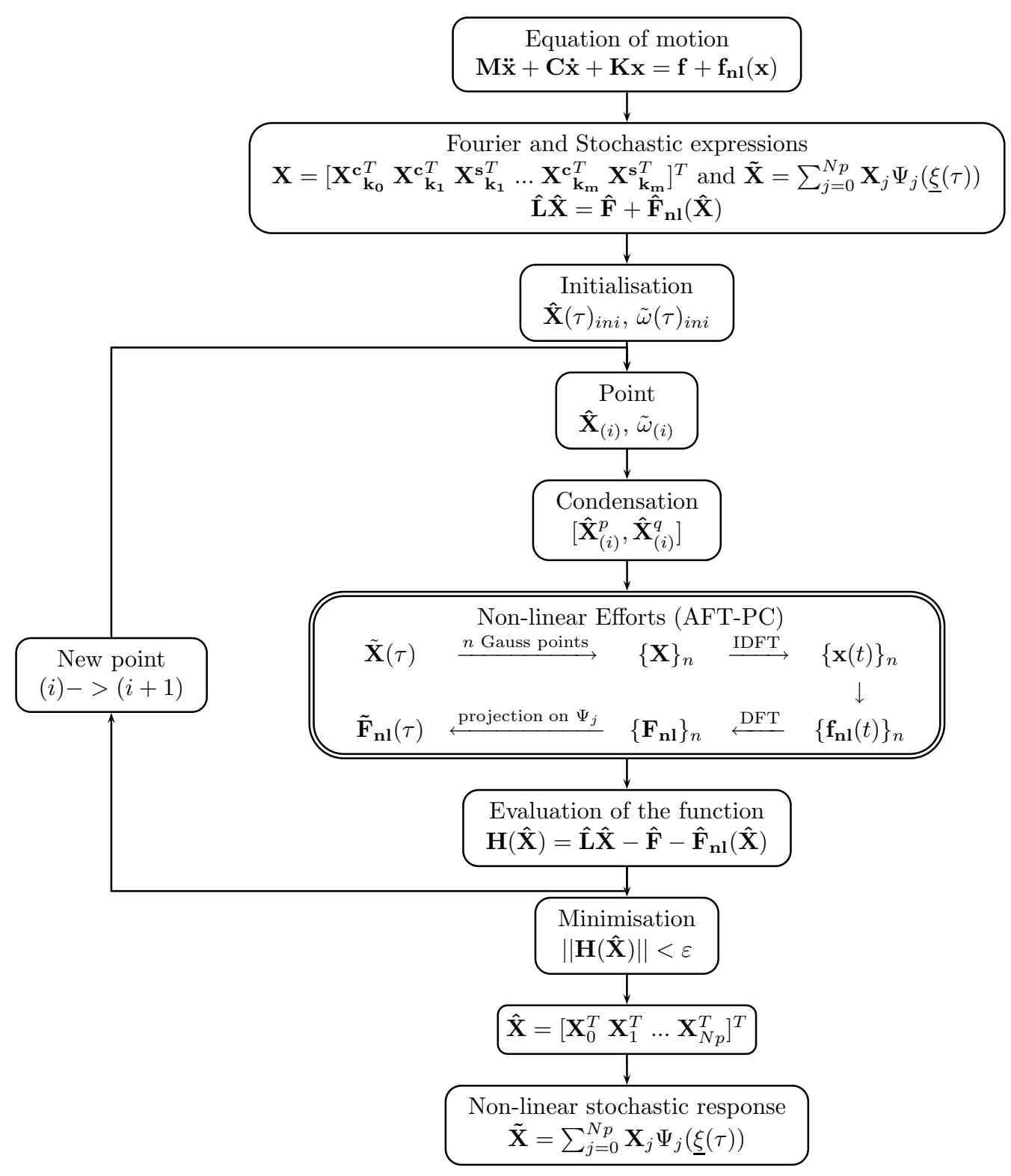

Fig. 2: General algorithm procedure for the Stochastic Multi-dimensional Harmonic Balance Method

where $\tau$ designates the random character. The dynamic equation of the system can be written in the stochastic domain as :

$$
\left(\tilde{\mathbf{I 0}}(\tau)+\omega_{1} \tilde{\mathbf{I}} \mathbf{1}(\tau)+\omega_{1}^{2} \tilde{\mathbf{I}}(\tau)\right) \tilde{\mathbf{X}}(\tau)=\tilde{\mathbf{F}}(\tau)+\tilde{\mathbf{F}_{\mathbf{n l}}}(\tau)
$$

with

$\tilde{\mathbf{I} 0}(\tau)=\operatorname{diag}\left(\tilde{\mathbf{K}}(\tau), \mathbf{I} \tilde{\mathbf{0}}_{1}(\tau), \ldots, \mathbf{I} \tilde{\mathbf{0}}_{m}(\tau)\right) \quad ; \quad \tilde{\mathbf{I}} \mathbf{1}(\tau)=\operatorname{diag}\left(\mathbf{0}, \tilde{\mathbf{I}}_{1}(\tau), \ldots, \tilde{\mathbf{I}}_{m}(\tau)\right) \quad ; \quad \tilde{\mathbf{I} 2}(\tau)=\operatorname{diag}\left(\mathbf{0}, \mathbf{I} \tilde{\mathbf{2}}_{1}(\tau), \ldots, \mathbf{\mathbf { I }} \tilde{\mathbf{2}}_{m}(\tau)\right)$

$\mathbf{I}_{j}(\tau)=\left[\begin{array}{cc}\tilde{\mathbf{K}}(\tau) & \mathbf{0} \\ \mathbf{0} & \tilde{\mathbf{K}}(\tau)\end{array}\right] \quad ; \quad \mathbf{I} \tilde{\mathbf{1}}_{j}(\tau)=\left[\begin{array}{cc}\mathbf{0} & \mathbf{\mathbf { k } _ { \mathbf { j } }} \cdot \boldsymbol{\alpha} \tilde{\mathbf{C}}(\tau) \\ -\mathbf{k}_{\mathbf{j}} \cdot \boldsymbol{\alpha} \tilde{\mathbf{C}}(\tau) & \mathbf{0}\end{array}\right] \quad ; \quad \mathbf{I}_{\mathbf{2}_{j}}(\tau)=\left[\begin{array}{cc}-\left(\mathbf{k}_{\mathbf{j}} \cdot \boldsymbol{\alpha}\right)^{2} \tilde{\mathbf{M}}(\tau) & \mathbf{0} \\ \mathbf{0} & -\left(\mathbf{k}_{\mathbf{j}} \cdot \boldsymbol{\alpha}\right)^{2} \tilde{\mathbf{M}}(\tau)\end{array}\right]$

Firstly, the uncertain material parameters and the uncertain forces are expanded using the Karhunen-Loeve expansion [15] with the Galerkin formulation of the finite element method [5] as following

$$
\tilde{\mathbf{Z}}(\tau)=\overline{\mathbf{Z}}+\sum_{l=1}^{L} \xi_{l}(\tau) \mathbf{Z}_{l}
$$


where $\tilde{\mathbf{Z}}(\tau)$ is $\tilde{\mathbf{M}}(\tau), \tilde{\mathbf{C}}(\tau), \tilde{\mathbf{K}}(\tau)$ or coefficients in the uncertain forces. Then, for each uncertain quantity, we have one or several independent Gaussian random variables $\left\{\xi_{l}(\tau)\right\}$. Finally, $\overline{\mathbf{Z}}$ designates the mean of quantity $\tilde{\mathbf{Z}}$ and $\mathbf{Z}_{l}$ is the $l$ th term of the Karhunen-Loeve expansion.

The stochastic dynamic response $\tilde{\mathbf{X}}(\tau)$ is expanded on the Polynomial Chaos basis [5]. This method is founded on the theory of the Homogeneous Chaos [16] that express uncertainties via orthogonal polynomials. Random response is given by

$$
\tilde{\mathbf{X}}(\tau)=\sum_{j=0}^{\infty} \mathbf{X}_{j} \Psi_{j}(\underline{\xi}(\tau))
$$

where $\Psi_{j}(\underline{\xi}(\tau))$ designates the polynomials of the basis with $\underline{\xi}(\tau)$ the random vector and $\mathbf{X}_{j}$ the deterministic coefficient to be found. In this study, the uncertain parameters will follow the Gaussian law, consequently the polynomials considered are the Hermite polynomials. If the positive value is required for the quantity then random quantity will be truncated to positive values. Besides, expression (29) will be truncated to a finite number $N_{p}$ for the numerical study. $N_{p}$ is the number of polynomials defined by $N p=\frac{(p+r) !}{p ! r !}$ where $r$ is the number of random variables and $p$ is the chaos order. The truncated form is given by

$$
\tilde{\mathbf{X}}(\tau)=\sum_{j=0}^{N p} \mathbf{X}_{j} \Psi_{j}(\underline{\xi}(\tau))
$$

In the following, we will retain the truncate form of the modeling for the expansions used on the uncertain parameters.

The Karhunen-Loeve expansion for all material quantities and for forces coefficients, given by Equation (28), introduced in a more global quantity $\bullet_{j}$ as $\mathbf{I} \mathbf{0}_{j}$ for the mass, $\mathbf{I} \mathbf{1}_{j}$ for the damping, $\mathbf{I} \mathbf{2}_{j}$ for the rigidity, or $\mathbf{F}_{j}$ and $\mathbf{F}_{\mathbf{n l} j}$ for the forces, and identified on the whole basis defined by vector $\underline{\xi}(\tau)$, yields the more global random quantity to be written as:

$$
\tilde{\bullet}_{j}(\tau)=\sum_{j=0}^{\infty} \bullet_{j} \Psi_{j}(\underline{\xi}(\tau))
$$

Quantity $\bullet_{j}$ will be detailed in the next sections for each uncertain quantity of the problem.

The expansion given in Equation (31) (and truncated to a finite number) alone is not sufficient to solve nonlinear problems which include returning point (i.e. phenomena of reversal curve of the Frequency Response Function). We then need to consider parameter $\tilde{\omega}_{1}$ as random and then expand this new unknown on the chaos basis. It will allow to describe the envelopes of the response of the system and especially in the vicinity of the returning points. Then, we propose the following expansion :

$$
\tilde{\omega}_{1}(\tau)=\sum_{j=0}^{N p} \omega_{1 j} \Psi_{j}(\underline{\xi}(\tau))
$$

where $\omega_{1 j}$ are the deterministic coefficients to find. It leads Equation (25) to be rewritten as following

$$
\begin{aligned}
\left(\sum_{i=0}^{N p} \mathbf{I}_{i} \Psi_{i}\right) & \left(\sum_{j=0}^{N p} \mathbf{X}_{j} \Psi_{j}\right)+\left(\sum_{i=0}^{N p} \mathbf{I}_{i} \Psi_{i}\right)\left(\sum_{j=0}^{N p} \mathbf{X}_{j} \Psi_{j}\right)\left(\sum_{k=0}^{N p} \omega_{1 k} \Psi_{k}\right) \\
+ & \left(\sum_{i=0}^{N p} \mathbf{I} \mathbf{2}_{i} \Psi_{i}\right)\left(\sum_{j=0}^{N p} \mathbf{X}_{j} \Psi_{j}\right)\left(\sum_{k=0}^{N p} \omega_{1 k} \Psi_{k}\right)\left(\sum_{l=0}^{N p} \omega_{1 l} \Psi_{l}\right)=\sum_{j=0}^{N p} \mathbf{F}_{j} \Psi_{j}+\sum_{j=0}^{N p} \mathbf{F}_{\mathbf{n} l_{j}} \Psi_{j}
\end{aligned}
$$

and which, when projecting on $\left\{\Psi_{m}\right\}_{m=0}^{N p}$ basis, is:

$$
\begin{aligned}
\sum_{i=0}^{N p} \sum_{j=0}^{N p}\left\langle\Psi_{i} \Psi_{j} \Psi_{m}\right\rangle \mathbf{I} \mathbf{0}_{i} \mathbf{X}_{j} & +\sum_{i=0}^{N p} \sum_{j=0}^{N p} \sum_{k=0}^{N p}\left\langle\Psi_{i} \Psi_{j} \Psi_{k} \Psi_{m}\right\rangle \mathbf{I} \mathbf{1}_{i} \mathbf{X}_{j} \omega_{1 k} \\
& +\sum_{i=0}^{N p} \sum_{j=0}^{N p} \sum_{k=0}^{N p} \sum_{l=0}^{N p}\left\langle\Psi_{i} \Psi_{j} \Psi_{k} \Psi_{l} \Psi_{m}\right\rangle \mathbf{I} \mathbf{2}_{i} \mathbf{X}_{j} \omega_{1 k} \omega_{1 l}=\left(\mathbf{F}_{m}+\mathbf{F}_{\mathbf{n l} m}\right)\left\langle\Psi_{m}^{2}\right\rangle
\end{aligned}
$$

for $m=0,1, \ldots, N p$ and where $\langle$.$\rangle is the mathematical expectation.$

Finally, we obtain the following nonlinear system to solve

$$
\hat{\mathbf{L}} \hat{\mathbf{X}}=\hat{\mathbf{F}}+\hat{\mathbf{F}}_{\mathbf{n l}}(\hat{\mathbf{X}})
$$

of size $(2 m+1) \times N p \times n_{D O F}$ and having $(2 m+2) \times N p \times n_{D O F}$ unknowns where

$$
\hat{\mathbf{L}}_{j m}=\sum_{i=0}^{N p}\left\langle\Psi_{i} \Psi_{j} \Psi_{m}\right\rangle \mathbf{I} \mathbf{0}_{i}+\sum_{i=0}^{N p} \sum_{k=0}^{N p}\left\langle\Psi_{i} \Psi_{j} \Psi_{k} \Psi_{m}\right\rangle \mathbf{I} \mathbf{1}_{i} \omega_{1 k}+\sum_{i=0}^{N p} \sum_{k=0}^{N p} \sum_{l=0}^{N p}\left\langle\Psi_{i} \Psi_{j} \Psi_{k} \Psi_{l} \Psi_{m}\right\rangle \mathbf{I} \mathbf{2}_{i} \omega_{1 k} \omega_{1 l} \quad j, m=0, \ldots, N_{p}
$$


and

$$
\begin{aligned}
& \hat{\mathbf{X}}=\left[\begin{array}{llll}
\mathbf{X}_{0}^{T} & \mathbf{X}_{1}^{T} & \ldots & \mathbf{X}_{N p}^{T}
\end{array}\right]^{T}
\end{aligned}
$$

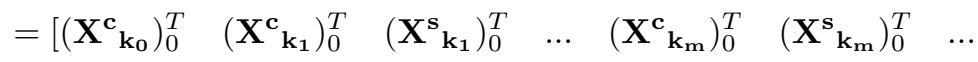

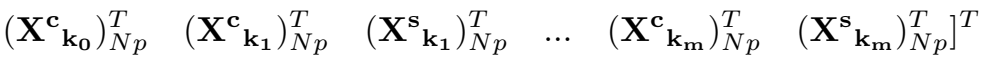

$$
\begin{aligned}
& \hat{\mathbf{F}}=\left[\begin{array}{llll}
\mathbf{F}_{0}^{T}\left\langle\Psi_{0}^{2}\right\rangle & \mathbf{F}_{1}^{T}\left\langle\Psi_{1}^{2}\right\rangle & \ldots & \mathbf{F}_{N p}^{T}\left\langle\Psi_{N p}^{2}\right\rangle
\end{array}\right]^{T}
\end{aligned}
$$

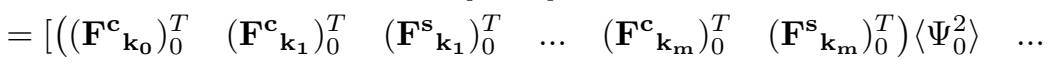

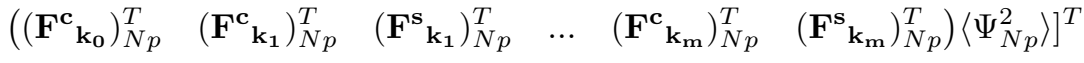

It should be noted that moments $\left\langle\Psi_{m}^{2}\right\rangle,\left\langle\Psi_{i} \Psi_{j} \Psi_{m}\right\rangle,\left\langle\Psi_{i} \Psi_{j} \Psi_{k} \Psi_{m}\right\rangle$ and $\left\langle\Psi_{i} \Psi_{j} \Psi_{k} \Psi_{l} \Psi_{m}\right\rangle$ can be computed by using a GaussHermite quadrature technique or analytically and only once for the whole problem. For the dynamic response and the linear forces components (i.e. $\left.\left(\mathbf{X}_{\mathbf{k}}^{\mathbf{c}}\right)_{m},\left(\mathbf{X}_{\mathbf{k}}^{\mathbf{s}}\right)_{m},\left(\mathbf{F}_{\mathbf{k}}^{\mathbf{c}}\right)_{m},\left(\mathbf{F}_{\mathbf{k}}^{\mathbf{s}}\right)_{m}\right)$, the subscript $\mathbf{k}$ refers to MHBM order and the second subscript $m$ refers to PCE order.

The nonlinear stochastic term $\hat{\mathbf{F}}_{\mathbf{n l}}$ is defined as

$$
\hat{\mathbf{F}}_{\mathbf{n l}}=\left[\begin{array}{llll}
\mathbf{F}_{\mathbf{n l}}^{T}\left\langle\Psi_{0}^{2}\right\rangle & \mathbf{F}_{\mathbf{n l}}^{T}\left\langle\Psi_{1}^{2}\right\rangle & \ldots & \mathbf{F}_{\mathbf{n l}}{ }_{N p}^{T}\left\langle\Psi_{N p}^{2}\right\rangle
\end{array}\right]^{T}
$$

where $\mathbf{F}_{\mathbf{n l} j}$ is evaluated via an Alternate Frequency-Time method with Probabilistic Collocation described in the following section.

To solve this nonlinear system of size $(2 m+1) \times N p \times n_{D O F}$ and having $(2 m+2) \times N p \times n_{D O F}$ unknowns, a phase condition between the stochastic response and the deterministic response is added. This condition can be formulated as following

$$
\sum_{\mathbf{k}=\mathbf{k}_{\mathbf{0}}}^{\mathbf{k}_{\mathbf{m}}} \operatorname{diag}\left(\mathbf{X}_{\mathbf{k}}^{\mathbf{c}} \otimes\left(\mathbf{X}_{\mathbf{k}}^{\mathbf{s}}\right)_{i}-\mathbf{X}_{\mathbf{k}}^{\mathbf{s}} \otimes\left(\mathbf{X}_{\mathbf{k}}^{\mathbf{c}}\right)_{i}\right)=\mathbf{0}
$$

for $i=0, \ldots, N_{p} . \otimes$ defines the tensorial product.

\subsubsection{Alternate Frequency-Time method with Probabilistic Collocation (AFT-PC method)}

As previously explained in Section 2.1, the Alternate Frequency Time (AFT) method allows to derive the Fourier components of the nonlinear forces as a function of the Fourier components of displacements in deterministic case. However, this procedure is not applicable in the case of stochastic problem with non regular nonlinearities due to the fact that it is not possible to obtain analytical expressions of the nonlinear forces in the stochastic domain neither in the time domain.

Consequently, it is necessary to calculate by another way than analytically unknown coefficients $\mathbf{F}_{\mathbf{n l} j}$ of Equation (31). The complete process named Alternate Frequency-Time method with Probabilistic Collocation to evaluate of the nonlinear force on the chaos basis and based on the combination of the Alternate Frequency Time and Polynomial Chaos methods is presented hereafter.

First of all, the unknown coefficients $\mathbf{F}_{\mathbf{n l} j}$ of Equation (31) are defined by

$$
\mathbf{F}_{\mathbf{n l} j}=\frac{\left\langle\tilde{\mathbf{F}_{\mathbf{n l}}}(\tau) \Psi_{j}(\underline{\xi})\right\rangle}{\left\langle\Psi_{j}^{2}(\underline{\xi})\right\rangle}
$$

which is in fact given by the inner product

$$
\left\langle a(\underline{\xi}) \Psi_{j}(\underline{\xi})\right\rangle=\int_{\Omega} a(\underline{\xi}) \Psi_{j}(\underline{\xi}) p(\underline{\xi}) d \underline{\xi}
$$

with $p(\underline{\xi})$ the probability density of $\underline{\xi}$.

This integral can be evaluated by the Probabilistic Collocation (PC) method using a Gauss quadrature [1] such as

$$
\left\langle a(\underline{\xi}) \Psi_{j}(\underline{\xi})\right\rangle=\sum_{i=1}^{n} w\left(\underline{\xi}_{i}\right) a\left(\underline{\xi}_{i}\right) \Psi_{j}\left(\underline{\xi}_{i}\right)
$$

where $\underline{\xi}_{i}$ are the Gaussian variables at collocation $i, n$ is the locations number and $w$ is the weight function defined, in the case of Gaussian random variables, by

$$
w(\underline{\xi})=\frac{1}{\sqrt{2 \pi}^{r}} e^{-\frac{\xi^{t} \xi}{2}}
$$


where $r$ is the vector size $\underline{\xi}$ that is the random variables number of the problem. Then Equation (41) is given by

$$
\mathbf{F}_{\mathbf{n l}_{j}}=\frac{\left\langle\tilde{\mathbf{F}}_{\mathbf{n l}}(\tau) \Psi_{j}(\underline{\xi})\right\rangle}{\left\langle\Psi_{j}^{2}(\underline{\xi})\right\rangle}=\frac{\sum_{i=1}^{n} w\left(\underline{\xi}_{i}\right) \tilde{\mathbf{F}}_{\mathbf{n l}}\left(\underline{\xi}_{i}\right) \Psi_{j}\left(\underline{\xi}_{i}\right)}{\sum_{i=1}^{n} w\left(\underline{\xi}_{i}\right) \Psi_{j}^{2}\left(\underline{\xi}_{i}\right)}
$$

So, we propose an extension of the Alternate Frequency Time method to evaluate the expressions of the nonlinear forces for stochastic problems. The methodology is based on the combination of the AFT and PC methods by using projection not only on the frequency basis but also on the chaos basis.

Let's start from $\tilde{\mathbf{X}}(\tau)$, the stochastic dynamic response of the nonlinear system (the size of the vector $\tilde{\mathbf{X}}(\tau)$ is $(2 m+1) \times$ $N_{p} \times n_{D O F}$ ), expanded on the Polynomial basis. Using, an IDFT (Inverse Discrete Fourier Transform) procedure, $n$ Gauss locations, one can evaluate the harmonic components of the response $\{\mathbf{X}\}_{n}$ in the frequency domain (the size of the vector $\{\mathbf{X}\}_{n}$ is $\left.(2 m+1) \times n_{D O F}\right)$. Then, one can express the associated displacements $\{\mathbf{x}(t)\}_{n}$ in the time-domain (the size of the vector $\{\mathbf{x}(t)\}_{n}$ is $n_{D O F}$ ) by using an IDFT (Inverse Discrete Fourier Transform) procedure. At this stage of process, we can note that the velocities $\{\dot{\mathbf{x}}(t)\}_{n}$ and accelerations $\{\ddot{\mathbf{x}}(t)\}_{n}$ of the response can also be calculated.

Then using the analytical expression of the nonlinear force (or a nonlinear operator) in time-domain (see expressions given in Equations (52), (53) and (54) as described in the next section for the current study), one can estimate the associated nonlinear expressions $\left\{\mathbf{f}_{\mathbf{n l}}(t)\right\}_{n}$ of the system (the size of the vector $\left\{\mathbf{f}_{\mathbf{n l}}(t)\right\}_{n}$ is $n_{D O F}$ ). Then, the DFT (Discrete Fourier Transform) algorithm allows the calculation of the harmonic components $\left\{\mathbf{F}_{\mathbf{n l}}\right\}_{n}$ of the nonlinear force in the frequency domain. Finally, the random vector $\tilde{\mathbf{F}}_{\mathbf{n l}}(\tau)$ for the nonlinear force can be obtained by the expansion on the chaos basis Equation (31) (i.e. $\tilde{\mathbf{F}_{\mathbf{n l}}}(\tau)=\sum_{j=0}^{N p} \mathbf{F}_{\mathbf{n l}} \Psi_{j}(\underline{\xi}(\tau))$ where $\Psi_{j}(\underline{\xi}(\tau))$ designates the polynomials of the basis). The size of the vector $\tilde{\mathbf{F}_{\mathbf{n l}}}(\tau)$ is $(2 m+1) \times N_{p} \times n_{D O F}$. The following diagram illustrates both the Alternate Frequency-Time method with Probabilistic Collocation:

$$
\begin{aligned}
& \tilde{\mathbf{X}}(\tau)=\sum_{j=0}^{N p} \mathbf{X}_{j} \Psi_{j}(\underline{\xi}(\tau)) \quad \underset{n \text { Gauss locations }}{\longrightarrow}\{\mathbf{X}\}_{n} \stackrel{\text { evaluation }}{\stackrel{\text { IDFT }}{\longrightarrow}}\{\mathbf{x}(t)\}_{n} \\
& \tilde{\mathbf{F}}_{\mathbf{n l}}(\tau)=\sum_{j=0}^{N p} \mathbf{F}_{\mathbf{n l} j} \Psi_{j}(\underline{\xi}(\tau)) \stackrel{\text { projection on } \Psi_{j}}{\longleftarrow} \quad\left\{\mathbf{F}_{\mathbf{n l}}\right\}_{n} \stackrel{\text { DFT }}{\longleftarrow}\left\{\mathbf{f}_{\mathbf{n l}}(t)\right\}_{n}
\end{aligned}
$$

It can be observed that the harmonic components of the response $\{\mathbf{X}\}_{n}$ in the frequency domain is an approximation of the "exact" value of $\mathbf{X}$ due to the evaluation via $n$ Gauss points. So, increasing the number of Gauss points allows a better estimation of the harmonic components of the response $\{\mathbf{X}\}_{n}$ in the frequency domain (i.e. a minimization of errors between the vector $\{\mathbf{X}\}_{n}$ and the unknown quantity $\left.\mathbf{X}\right)$. Moreover, it can be noted that the vector of displacements $\{\mathbf{x}(t)\}_{n}$ and the vector of the nonlinear forces $\left\{\mathbf{f}_{\mathbf{n l}}(t)\right\}_{n}$ in the time-domain and the harmonic components $\left\{\mathbf{F}_{\mathbf{n l}}\right\}_{n}$ of the nonlinear force in the frequency domain are also approximated expressions of the "exact" values of $\mathbf{x}(t), \mathbf{f}_{\mathbf{n l}}(t)$ and $\mathbf{F}_{\mathbf{n l}}$ due to the previous process.

\subsubsection{Condensation process for the stochastic nonlinear problem}

As previously explained by Sinou [17, 14], if a nonlinear deterministic system consists of a $n$-degree-of-freedom system with nonlinear forces associated with $q$ of these degrees-of-freedom, it may be of great interest to keep only the $q$ nonlinear degrees-offreedom. The classical procedures of condensation on the nonlinear degrees-of-freedom developed to treat this type of problem are still applicable to the stochastic problem. So, the previous nonlinear Equation (35) is transformed by

$$
\left[\begin{array}{cc}
\hat{\mathbf{L}}_{l n, l n} & \hat{\mathbf{L}}_{l n, n l} \\
\hat{\mathbf{L}}_{n l, l n} & \hat{\mathbf{L}}_{n l, n l}
\end{array}\right]\left(\begin{array}{c}
\hat{\mathbf{X}}_{l n} \\
\hat{\mathbf{X}}_{n l}
\end{array}\right)=\left(\begin{array}{c}
\hat{\mathbf{F}}_{l n} \\
\hat{\mathbf{F}}_{n l}
\end{array}\right)+\left(\begin{array}{c}
\mathbf{0} \\
\hat{\mathbf{F}}_{\mathbf{n l}}
\end{array}\right)
$$

where $\hat{\mathbf{X}}_{l n}$ and $\hat{\mathbf{X}}_{n l}$ contain the $n-q$ linear degrees-of-freedom and the $q$ nonlinear degrees-of-freedom, respectively. Here, we do not present the condensation procedure used to obtain the Fourier coefficients associated with the nonlinear and linear elements of the complete system. We refer the interested reader to the following paper [14] for more details.

After calculations, the determination of the Fourier components $\hat{\mathbf{X}}_{n l}$ associated with the nonlinear elements can be obtained by considering the following condensed system

$$
\hat{\mathbf{L}}_{e q} \hat{\mathbf{X}}_{n l}=\hat{\mathbf{F}}_{n l}+\hat{\mathbf{F}}_{e q}
$$

with

$$
\hat{\mathbf{L}}_{e q}=\hat{\mathbf{L}}_{n l, n l}-\hat{\mathbf{L}}_{n l, l n} \hat{\mathbf{L}}_{l n, l n}^{-1} \hat{\mathbf{L}}_{l n, n l} \quad ; \quad \hat{\mathbf{F}}_{e q}=\hat{\mathbf{F}}_{\mathbf{n l}}-\hat{\mathbf{L}}_{n l, l n} \hat{\mathbf{L}}_{l n, l n}^{-1} \hat{\mathbf{F}}_{l n}
$$

It should be noted that for a general nonlinear system, certain linear degrees-of-freedom of the vector $\hat{\mathbf{X}}_{l n}$ can be transferred to the vector $\hat{\mathbf{X}}_{n l}$ of the nonlinear degree-of-freedom without loosing the general process presented previously. This operation can be very interesting if keeping the physical linear degree-of-freedom is necessary for the study.

Moreover, the same condensation process can be applied to the linear elements. After calculation, we obtain the following relations

$$
\hat{\mathbf{L}}_{l n, l n} \hat{\mathbf{X}}_{l n}=\hat{\mathbf{F}}_{l n}-\hat{\mathbf{L}}_{l n, n l} \hat{\mathbf{X}}_{n l}
$$




\section{Numerical studies}

In this section, in order to verify the suitability of the proposed approach, we will present numerical example cases for a nonlinear two-degrees-of-freedom model with different types of nonlinearities and uncertainties.

\subsection{Description of the nonlinear model under study}

Figure 3 shows the nonlinear two-degrees-of-freedom model to be used in the following. This minimal two-degrees-of-freedom model is chosen due to its simplicity and to better understand the effects of uncertainties of various physical parameters including, more specifically, the nonlinear elements of the system.

The equations of motion take the following form for this system:

$$
\left[\begin{array}{cc}
m & 0 \\
0 & m
\end{array}\right]\left(\begin{array}{c}
\ddot{x}_{1} \\
\ddot{x}_{2}
\end{array}\right)+\left[\begin{array}{cc}
2 c & -c \\
-c & 2 c
\end{array}\right]\left(\begin{array}{c}
\dot{x}_{1} \\
\dot{x}_{2}
\end{array}\right)+\left[\begin{array}{cc}
2 k & -k \\
-k & 2 k
\end{array}\right]\left(\begin{array}{c}
x_{1} \\
x_{2}
\end{array}\right)=\left(\begin{array}{c}
f_{1} \cos \left(\omega_{1} t\right) \\
f_{2} \sin \left(\omega_{2} t\right)
\end{array}\right)+\left(\begin{array}{c}
f_{n l_{1}} \\
f_{n l_{2}}
\end{array}\right)
$$

Two cases will be investigated for the excitation of the system. The first one considers only one excitation of frequency $\omega_{1}$ (with $f_{2} \sin \left(\omega_{2} t\right)=0$ ). So the response of the nonlinear system is represented as truncated Fourier series with $\omega_{1}$ the fundamental frequency. The second one considers that the global excitation of the system is composed by two external incommensurable frequencies $\omega_{1}$ et $\omega_{2}$ with

$$
\frac{\omega_{2}}{\omega_{1}}=\frac{1}{\sqrt{2}}
$$

Therefore the vector of the frequency basis is given by $\boldsymbol{\omega}=\left[\begin{array}{ll}\omega_{1} & \omega_{2}\end{array}\right]^{T}$ and $\mathbf{k}=\left[k_{1}, k_{2}\right]$. So the bi-periodic motion of the nonlinear system is an equivalent function of 2 time variables in a 2-dimensional time domain and it covers the invariant torus in the phase space $[7,12]$.

$f_{n l_{1}}$ and $f_{n l_{2}}$ define the nonlinear contributions applied to the mechanical system. In the present study, various numerical examples will be conducted. These cases were chosen to demonstrate the robustness and efficiency of the stochastic nonlinear method with respect to various types of nonlinearity (regular or non-regular).

The first one consider a cubic polynomial nonlinearity (see Figure 3(c)) given by

$$
f_{n l_{1}}(t)=-k_{n l} x_{1}^{3}(t)
$$

where $k_{n l}$ defines the cubic nonlinear term and $x_{1}(t)$ is the temporal displacement for the first degree-of-freedom.

Secondly, a non-regular nonlinearity with contact to no-contact states is investigated (see Figure 3(d)). The restoring force can be estimated by the following relation

$$
\left\{\begin{array}{l}
f_{n l_{1}}(t)=-k_{1} x_{1}(t) \quad \text { if } \quad\left|x_{1}(t)\right| \leq x_{l i m} \\
f_{n l_{1}}(t)=-k_{2} x_{1}(t)+\operatorname{sign}\left(x_{1}(t)\right)\left(k_{2}-k_{1}\right) x_{l i m} \quad \text { if } \quad\left|x_{1}(t)\right|>x_{l i m}
\end{array}\right.
$$

where $k_{1}$ and $k_{2}$ define effective stiffnesses and $x_{\text {lim }}$ represents the clearance value.

The last case considers the rubbing phenomena with friction dissipation mechanism and stick-slip motion [18, 19], as illustrated in Figures 3(b) and (e). The nonlinear forces $f_{n l_{1}}(t)$ due to friction is expressed, according to the Coulomb law, as:

$$
\left\{\begin{array}{l}
f_{n l_{1}}(t)=-k_{f}\left(x_{1}(t)-z(t)\right) \quad \text { if adhesion state } \\
f_{n l_{1}}(t)=-\mu P \operatorname{sign}(\dot{z}(t)) \quad \text { if slip state }
\end{array}\right.
$$

where $k_{f}$ defines the contact stiffness, $\mu$ the constant friction coefficient, $P$ the normal contact load, and $z(t)$ is the relative displacement of the contact point.

The values of the physical parameters are given in Table 1.

\subsection{Uncertainties in system}

The uncertain parameters considered in the problem are the following. As mentioned previously, they are expanded in a Karhunen-Loeve expansion (Equation (28)) and the random variables are taken all as Gaussian variables.

First, the linear stiffness is taken as random and is defined as

$$
\tilde{k}=\bar{k}\left(1+\delta_{k} \xi_{1}\right)
$$

where $\xi_{1}$ is one Gaussian random variable. Quantity $\delta_{k}$ designates the variation coefficient of the random stiffness. 


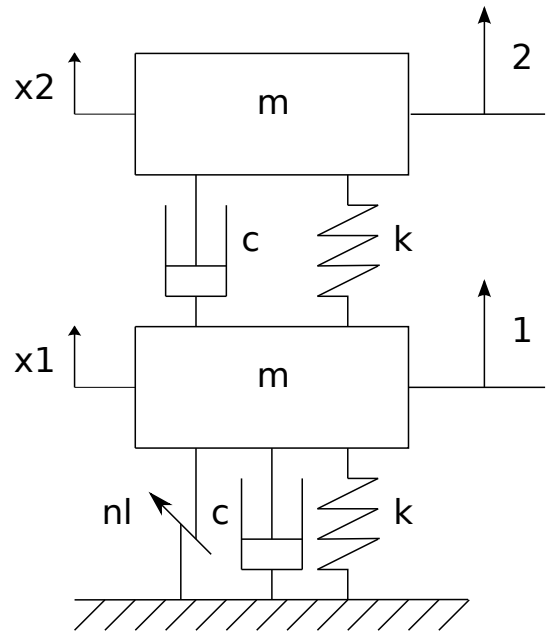

(a)

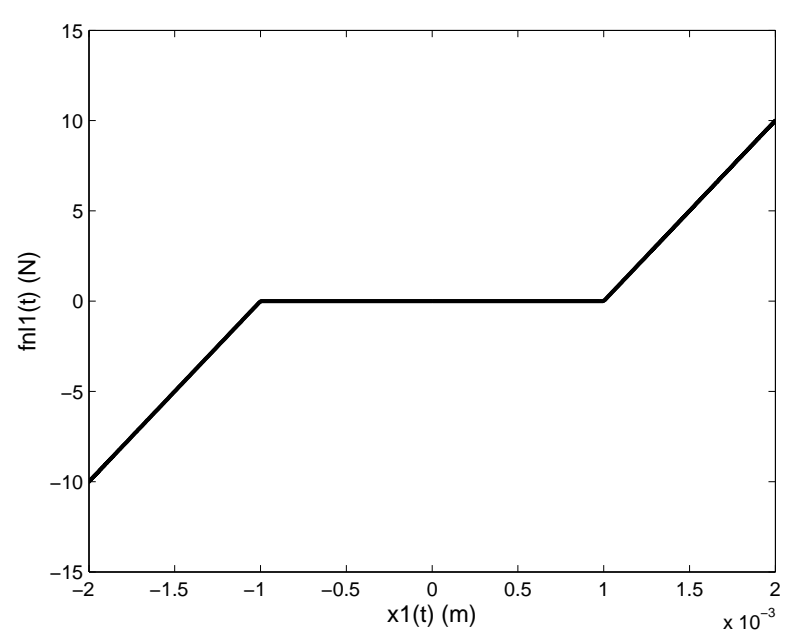

(d)

(b)
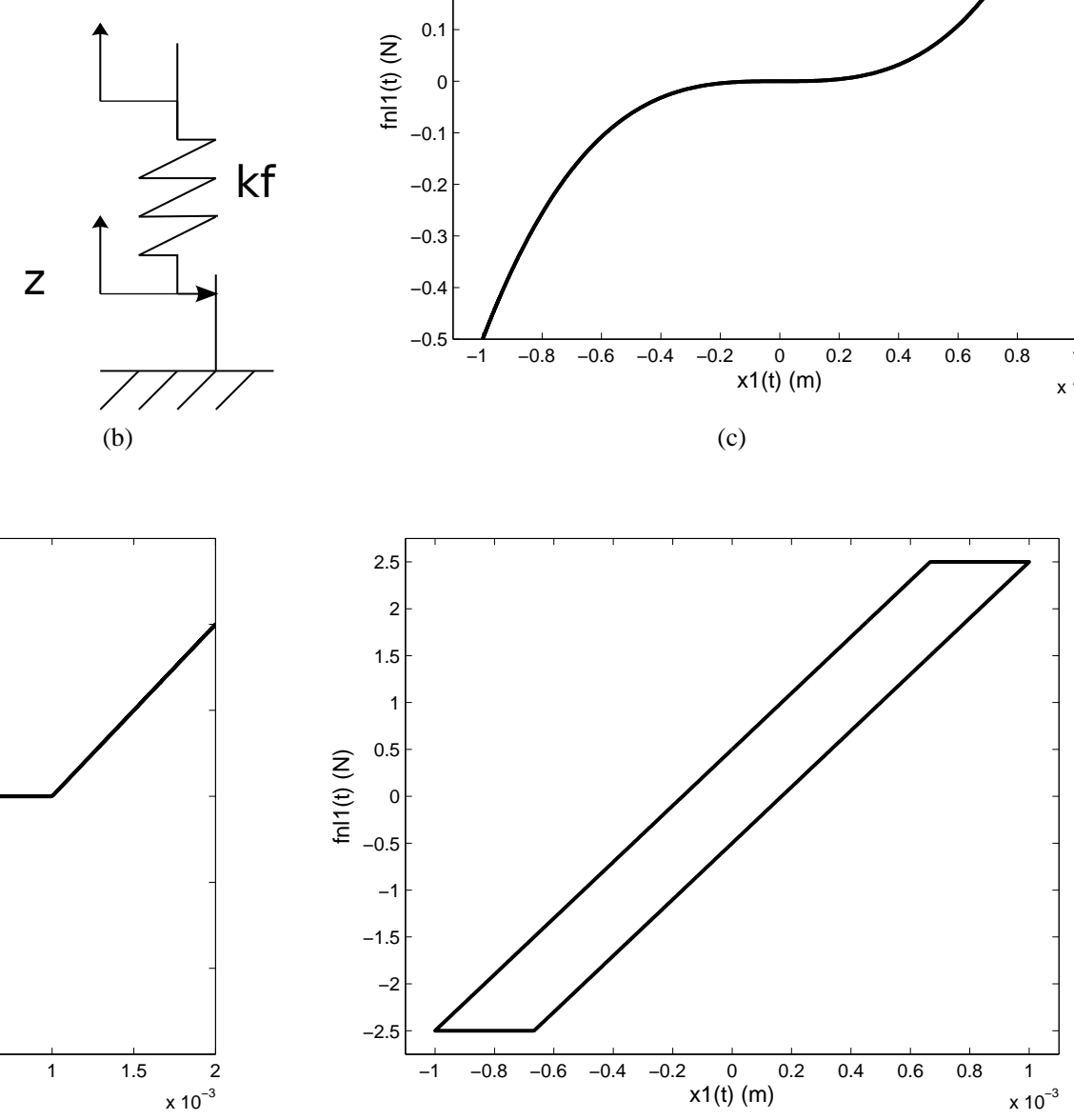

(e)

Fig. 3: Two-degrees-of-freedom model with nonlinearities (a) 2 DOFs model (b) friction model (c) cubic stiffness; (d) contact; (e) friction.

To validate the efficiency of the stochastic nonlinear method, the nonlinear parts of the two degree-of-freedom system will also be random. For the first type of nonlinearity (cubic nonlinearity, see Figure 3(c)), we define a random nonlinear stiffness $\tilde{k}_{n l}$ following:

$$
\tilde{k}_{n l}=\overline{k_{n l}}\left(1+\delta_{k_{n l}} \xi_{2}\right)
$$

For the second type of nonlinearity (contact and no-contact states, see Figure 3(d)), we consider the parameter $\tilde{k}_{2}$ that defines the effective stiffness of the system in contact state as stochastic:

$$
\tilde{k}_{2}=\overline{k_{2}}\left(1+\delta_{k_{2}} \xi_{3}\right)
$$

Finally, in the frictional case (third nonlinear case, Figure 3(e)), the random parameter which seems the most interesting is the friction coefficient $\mu$. Indeed, this parameter can usually be unknown due to various operating conditions or tribological phenomena such as wear. Then, we can expand this parameter as:

$$
\tilde{\mu}=\bar{\mu}\left(1+\delta_{\mu} \xi_{4}\right)
$$




\begin{tabular}{ll}
\hline Parameter & Value \\
\hline$k$ & $15000 \mathrm{~N} \cdot \mathrm{m}^{-1}$ \\
$m$ & $1 \mathrm{~kg}$ \\
$c$ & $1 \mathrm{~N} \cdot \mathrm{m}^{-1} \cdot \mathrm{s}^{-1}$ \\
$f_{1}$ & $1 \mathrm{~N}$ \\
$f_{2}$ & $1 \mathrm{~N}$ \\
$k_{f}$ & $3000 \mathrm{~N} \cdot \mathrm{m}^{-1}$ \\
$P$ & $10 \mathrm{~N}$ \\
$k_{1}$ & $5.10^{-4} \mathrm{~N} \cdot \mathrm{m}^{-1}$ \\
$k_{2}$ & $5.10^{3} \mathrm{~N} \cdot \mathrm{m}^{-1}$ \\
\hline
\end{tabular}

Tab. 1: Parameter values

\begin{tabular}{lllllllllll}
\hline Case & $\delta_{k}$ & $\overline{k_{n l}}\left(\mathrm{~N} \cdot \mathrm{m}^{-\frac{1}{3}}\right)$ & $\delta k_{n l}$ & $\overline{k_{2}}\left(\mathrm{~N} \cdot \mathrm{m}^{-1}\right)$ & $\delta k_{2}$ & $\bar{\mu}$ & $\delta_{\mu}$ & HBM order & HBM dim & PCE order \\
\hline 1 & $2.5 \%$ & $5.10^{8}$ & - & - & - & - & - & 3 & 1 & 3 \\
2 & - & $5.10^{8}$ & $10 \%$ & - & - & - & - & 3 & 1 & 3 \\
$2 \mathrm{bis}$ & - & $5.10^{9}$ & $10 \%$ & - & - & - & - & 3 & 1 & 3 \\
2 ter & - & $5.10^{10}$ & $10 \%$ & - & - & - & - & 3 & 1 & 3 \\
3 & - & - & - & $5.10^{3}$ & $5 \%$ & - & - & 1 & 1 & 3 \\
4 & - & - & - & - & - & 0.3 & $5 \%$ & 1 & 1 & 3 \\
$4 \mathrm{bis}$ & - & - & - & - & - & 0.4 & $5 \%$ & 1 & 1 & 3 \\
4 ter & - & - & - & - & - & 0.5 & $5 \%$ & 1 & 1 & 3 \\
5 & $2.5 \%$ & - & - & $5.10^{3}$ & $5 \%$ & - & - & 1 & 1 & 3 \\
6 & $2.5 \%$ & - & - & $5.10^{3}$ & - & - & - & 1 & 2 & 3 \\
7 & - & - & - & - & - & 0.4 & $5 \%$ & 1 & 2 & 3 \\
\hline
\end{tabular}

Tab. 2: Sets of parameters for the case studies

\subsection{Results}

In this section, the relevance of the proposed Stochastic Multi-dimensional Harmonic Balance Method is demonstrated through different sets of parameters for which uncertainty values are given in Table 2. Information concerning the order of chaos, the order of the truncated Fourier series and the type of nonlinearities are also given in this table.

Regarding the types of excitation applied to the two-degree-of-freedom system, we will first treat the case of mono-frequency excitation $\omega_{1}$ (for cases 1, 2, 2bis, 2ter, 3, 4, 4bis, 4ter and 5) and then two cases (case 6 and 7) with two external incommensurable frequencies $\omega_{1}$ et $\omega_{2}$ (with $\frac{\omega_{2}}{\omega_{1}}=\frac{1}{\sqrt{2}}$ ). All the results are presented for the second degree-of-freedom $x_{2}$. For the case with a mono-frequency excitation, the general Stochastic Multi-dimensional Harmonic Balance Method will be restricted to the Stochastic Harmonic Balance Method that corresponds to the simplification of the Stochastic-MHBM with only one-dimensional excitation.

To validate the Stochastic Multi-dimensional Harmonic Balance Method, all the calculations are compared to those obtained from the combination of the classical deterministic Multi-dimensional Harmonic Balance Method that has been previously validated in $[10,14]$ and the Monte Carlo simulations. Figure 4 represents the global computational procedure for both the Monte Carlo simulations and the Stochastic Multi-dimensional Harmonic Balance Method. For the reference solutions $\{\mathbf{X}\}_{i(\mathrm{MCS})}$ (for $i=1, \ldots, N)$ computed from the Monte Carlo simulations with the deterministic Multi-dimensional Harmonic Balance Method, $N$ samples of the random vector $\{\underline{\xi}(\tau)\}_{i}$ (for $i=1, \ldots, N$ ) are considered. The number of samples $N$ is taken equal to 1000 for the present study. As indicated in Figure 4, the results obtained via of the Stochastic Multi-dimensional Harmonic Balance Method that are expressed on the basis of chaos are post-processed using the formulation of the truncated stochastic dynamic response $\tilde{\mathbf{X}}(\tau)$ given in Equation (30). The same samples $\{\underline{\xi}(\tau)\}_{i}$ are then used for the evaluation of Hermite polynomials as indicated in Figure 4. This allows us to obtain the nonlinear solutions $\{\tilde{\mathbf{X}}\}_{i \text { (Stochastic-HBM) }}$ (for $i=1, \ldots, N$ ) that will be compared to the previous reference solutions $\{\mathbf{X}\}_{i(M C S)}$. We recall that the stochastic dynamic response $\tilde{\mathbf{X}}(\tau)$ given in Equation (30) has been previously calculated via the Stochastic Multi-dimensional Harmonic Balance Method without specification of these samples.

For all results, the order of chaos is set at 3 due to the fact that preliminary studies, not presented here, for various orders of chaos led to the conclusion that an order 3 is sufficient for each case. 


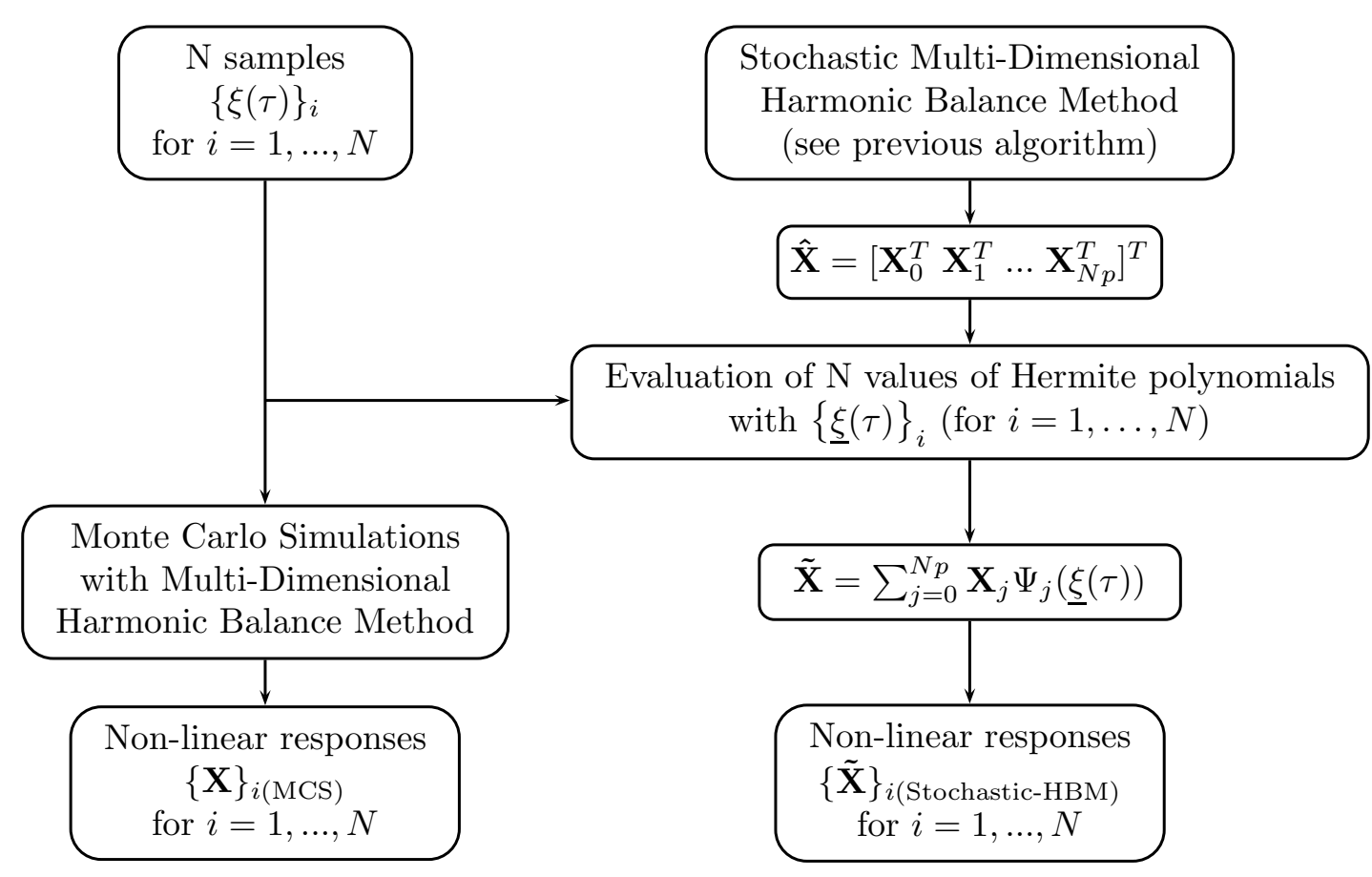

Fig. 4: Computational procedure for both the Monte Carlo simulations and the Stochastic Multi-dimensional Harmonic Balance Method

\subsubsection{Case 1: uncertainty in the linear stiffness}

For case 1 , we consider a random variation of $\delta_{k}=2.5 \%$ for the stiffness properties of the mechanical system (as indicated in Equation (55)). All the other physical parameters are kept deterministic and a mono-excitation of frequency $\omega_{1}$ is applied on the nonlinear system. The nonlinear term is chosen of type cubic stiffness contribution in this first case. Figures 5 show the Frequency Response Functions for the random global nonlinear response and the $n \times$ super-harmonic component (for $n=1$ and $n=3$ ) for both Monte-Carlo Simulation and the Stochastic Harmonic Balance Method. A very good agreement is obtained between the results from the MCS and the Stochastic-HBM. As expected, the random parameter influences not only the $1 \times$ response (see Figures $5(\mathrm{c}, \mathrm{d})$ ), but also the $3 \times$ response (see Figures $5(\mathrm{e}, \mathrm{f})$ ). Even if uncertainty is introduced initially into one linear term (i.e. linear stiffness of the system), due to the nonlinear behavior of the system and the coupling between the linear contribution and the nonlinear operator defined in Equation (52), uncertainty is also found on the nonlinear terms via the Alternate Frequency-Time method with Probabilistic Collocation. Moreover, this first example illustrates the fact that the Stochastic Harmonic Balance Method is able to predict variations of the global nonlinear response and the $n \times$ super-harmonic components around the reversal curve of the Frequency Response Function (between $12 \mathrm{~Hz}$ and $14 \mathrm{~Hz}$ as shown in Figures 5(a,b) for the global nonlinear response and in Figures 5(c,d) and (e,f) for the $1 \times$ and $3 \times$ super-harmonic components, respectively). It is recalled that this phenomena is only due to the hardening effect of the nonlinearity. This result of monitoring the evolution of the random nonlinear response around the turning point is possible due to the fact that the pulsation frequency is considered to be random, as previously introduced in Equation (32).

\subsubsection{Cases 2, 2bis and 2ter: uncertainty in the nonlinear cubic stiffness}

In this subsection, we are interested in the effect of variations on the cubic stiffness. We focus our attention on three cases with different values of the mean cubic stiffness $\overline{k_{n l}}$ (case 2: $\overline{k_{n l}}=5.10^{8} \mathrm{~N} \cdot \mathrm{m}^{-\frac{1}{3}}$; case $2 \mathrm{bis:} \overline{k_{n l}}=5.10^{9} \mathrm{~N} \cdot \mathrm{m}^{-\frac{1}{3}}$ and case 2ter: $\overline{k_{n l}}=5.10^{10} \mathrm{~N} \cdot \mathrm{m}^{-\frac{1}{3}}$ ). All the other physical parameters are kept deterministic and a mono-excitation of frequency $\omega_{1}$ is applied on the nonlinear system. It is recalled that increasing the nonlinear cubic stiffness increases the hardening behavior of the mechanical system (i.e. the Frequency Response Function shows a more curved peak frequency and a more abrupt turning point). Variation of $\delta_{k_{n l}}=10 \%$ on the cubic term is considered (as previously introduced in Equation (56)).

Figures 6 present the results obtained by the Stochastic-HBM and MCS approaches for the three cases (note that each curve is indicated by the number of the associated cases 2, 2bis or 2ter in Figures 6(a) and 6(b)). In all cases, the nonlinear behavior is mainly due to first harmonic component even if the $3 \times$ harmonic component is visible around $5 \mathrm{~Hz}$ for case 2ter (with $\overline{k_{n l}}=5 \cdot 10^{10} \mathrm{~N} \cdot \mathrm{m}^{-\frac{1}{3}}$ ). Moreover, Figure 6(d) clearly indicates that the $3 \times$ harmonic component is not negligible: we can see the appearance of peaks with more or less strong hardening effect for cases 2, 2bis or 2ter. The results obtained by MCS approach for the $1 \times$ and $3 \times$ harmonic components are not presented in this paper due to the fact that they are similar to those obtained by the Stochastic-HBM (given in Figures 6(c) and 6(d)). 
Then, Figure 7 shows a zoom of the evolutions of the random response of the $1 \times$ and $3 \times$ harmonic component around the first frequency peak (between $10 \mathrm{~Hz}$ and $16 \mathrm{~Hz}$ ). Some samples computed from the MCS and Stochastic-HBM are shown in Figure 7. We recall that the Stochastic-HBM results that are expressed on the basis of chaos are post-processed using the formulation of the truncated stochastic dynamic response given in Equation (30)) with the samples previously used by the Monte-Carlo simulations. The remarks previously stated remain valid: a perfect correlation between the Stochastic-HBM and MCS results (for each random solution of the $n \times$ harmonic components) is observed even if the hardening effect is very strong. These results clearly demonstrate the efficiency of the Stochastic-HBM.

Finally, as illustrated in Figures 6 and 7, the response of the nonlinear system is not unique for some excitation frequencies. Over a range of frequencies near the resonance, the system can take one of three possible response levels. For example, three possible nonlinear responses with low amplitude or possible high amplitude coexist in the intervals $[13 ; 17] \mathrm{Hz}$ and $[33 ; 55] \mathrm{Hz}$ for a given frequency. Here, we clearly show that the Stochastic-HBM approach is able to find the nonlinear solution in the vicinity of the initial conditions (given by the previous calculation). So, all the FRF curve with low and high amplitude responses can be depicted by the Stochastic-HBM approach. Moreover, we see the evolution of the second resonance peak to the right due to uncertainty in the nonlinear cubic stiffness. As previously explained, this phenomena reflects the fact that a variation of the nonlinear cubic stiffness induces not only an evolution of the nonlinear response amplitudes but also a variation of the fundamental frequency of the nonlinear system. This is one of the most difficult aspects to be captured by uncertainty methods being applied to nonlinear systems. Here, it can be observed that the Stochastic-HBM approach is able to find the response amplitudes due to the fact that the pulsation frequency is considered to be random, as proposed in Equation (32). All this results allows us to conclude on the robustness of the global Stochastic-HBM approach with the Alternate Frequency-Time method with Probabilistic Collocation.

\subsubsection{Case 3: uncertainty in the contact nonlinear stiffness}

For case 3, the nonlinear term is chosen to be the contact/non-contact behavior and we are interested in the effect of a variation on this nonlinear element of $\delta_{k_{2}}=5 \%$ (Equation (57)). All the other physical parameters are kept deterministic and a monoexcitation of frequency $\omega_{1}$ is applied on the nonlinear system.

Figures 8(a) presents the results obtained by the Stochastic-HBM approach. Zooms near the first and second resonance peaks are illustrated in Figures 8(b) by applying the Stochastic-HBM and MCS approaches. As previously indicated in section 3.3.2, only some samples computed from the MCS and Stochastic-HBM are compared. The remarks previously stated remain valid: a perfect correlation between the Stochastic-HBM and MCS results (for each random solution of the $n \times$ harmonic components) is observed even if the hardening effect is very strong. These results clearly demonstrate the efficiency of the Stochastic-HBM.

These comparisons allow us to validate the methodology proposed in this study. As previously explained, we observed that a variation of the contact nonlinear stiffness induces an evolution of the resonance frequency.

In the vicinity of the resonance peaks, one observes the classical behavior and characteristic of nonlinear systems with contact: a break of slope of the resonance peak is observed due to the transition between the non contact state and contact state (see Figures 8(b) for example). Moreover, variation of the contact stiffness induces a variability of the resonance frequency and a shift of the resonance peaks to the right. Here again, using the Stochastic-HBM approach, it is possible to describe the multiple nonlinear solutions with low or high amplitudes in the interval between $[12.3 ; 13.3] \mathrm{Hz}$ (see Figure 8(b), top figure) and $[31.7 ; 31.9] \mathrm{Hz}$ (see Figure 8(b), bottom figure) for a given frequency. This demonstrates once again the effectiveness and robustness of the proposed Stochastic-HBM approach.

\subsubsection{Cases 4, 4bis and 4ter: uncertainty in the friction element}

In this subsection, we focus our attention on the two-degree-of-freedom system with a the friction nonlinear element. As previously explained, one of the most interesting parameter to be random is the friction coefficient $\mu$. So we investigate the effect of variations on the friction coefficient $\delta_{\mu}=5 \%$ on three cases with different values of the mean friction coefficient $\bar{\mu}$ (case 4 : $\bar{\mu}=0.3$; case 4bis: $\bar{\mu}=0.4$ and case 4ter: $\bar{\mu}=0.5$ ). All the other physical parameters are kept deterministic and a monoexcitation of frequency $\omega_{1}$ is applied on the nonlinear system.

Figures 9 illustrated the Frequency Response Functions of the random global nonlinear response for cases 4, 4bis and 4ter by applying the Stochastic-HBM. Zooms of the random nonlinear responses (for only thirty selected samples) around the first and second resonance peaks $([11.5 ; 14] \mathrm{Hz}$ and $[31 ; 34] \mathrm{Hz}$, respectively) for both the Stochastic-HBM and MCS methods are plotted in Figures $9(\mathrm{~b}, \mathrm{c}, \mathrm{d})$ for different mean friction coefficients with the same uncertainty quantity $\delta_{\mu}=5 \%$. By comparing the Stochastic-HBM results with those estimated by Monte Carlo Simulations, we can first notice that the efficiency of the StochasticHBM approach is validated in the case of a nonlinear frictional element.

Then, we note the global progressive frequency shift of the resonance peaks due to the mean friction coefficient with the additional uncertainty quantity $\delta_{\mu}=5 \%$ (cases 4 to 4 ter). This phenomenum reflects the fact the nonlinear effect of friction has usually a softening effect on system dynamic.

So it can be concluded that all these results are consistent with the trends already known on the dissipative effects due to friction. These cases also demonstrate the possibility of including uncertainties aspects to better characterize the dynamic behavior of nonlinear systems with friction by using the Stochastic-HBM method. 


\subsubsection{Case 5: uncertainties for both the linear stiffness and the contact nonlinear stiffness}

In order to demonstrate the efficiency of the Stochastic-HBM procedure described above, this subsection of the paper treats the cases in which uncertain quantities come from two parameters studied previously: the first one concerns the stiffness with the mean $\bar{k}=15000 \mathrm{Nm}^{-1}$ and a variation coefficient of $\delta_{k}=2.5 \%$. The second uncertain quantity is the stiffness of the contact/non-contact element with the mean stiffness $\overline{k_{2}}=5000 \mathrm{Nm}^{-1}$ and a variation coefficient of $\delta_{k_{2}}=5 \%$. A monoexcitation of frequency $\omega_{1}$ is applied on the nonlinear system.

We recall that in this case the cost of calculation may be greater than in the previous cases since it is directly linked to the number of polynomials and consequently to the order of chaos and the number of random parameters.

Figures 10 illustrate all the Frequency Response Function samples for the nonlinear response amplitudes obtained by using the MCS and Stochastic-HBM approaches. The results from both methods are in very good agreement. As explained previously in sections 3.3.1 and 3.3.3, variations of physical parameters leads to changes for both the resonance peak frequencies and the dynamic response of the system around the peak frequencies.

In comparison with the previous results obtained in sections 3.3.3 and illustrated in Figures 8, it can be seen that the effects on the response from uncertainties on the linear stiffness are greater than those on the effective stiffness of the contact for the values of physical parameters given in Table 2 for cases 3 and 5 . So even if the variation coefficient of the effective contact stiffness $\delta_{k_{2}}$ is greater than the linear stiffness $\delta_{k}$, it may be concluded that the variations of nonlinear amplitudes are mainly due to the effects of uncertainty on the linear stiffness. However, whatever the levels of uncertainty, it is recalled that the variation coefficient of $\delta_{k}$ affects also the nonlinear response of the system, as previously demonstrated in section 3.3.3.

In conclusion, this example demonstrated the robustness of the Stochastic-HBM approach when uncertain quantities come from two parameters.

\subsubsection{Case 6: uncertainty in the linear stiffness with incommensurable multi-excitations}

For this case, the two-degree-of-freedom system is subjected to two external incommensurable frequencies $\omega_{1}$ et $\omega_{2}$ (with $\frac{\omega_{2}}{\omega_{1}}=\frac{1}{\sqrt{2}}$ ). The two-degree-of-freedom has a deterministic effective contact stiffness (i.e. $k_{2}=5.10^{3} \mathrm{Nm}-1$ ) and a variation coefficient of $\delta_{k}=2.5 \%$ for the stiffness of the mechanical system.

At first, we propose to focus on the overall quasi-periodic response of the system. Figure 11(a) and 11(b) illustrate the global nonlinear amplitudes obtained by using the MCS and the Stochastic-MHBM, respectively. We can note an important variation of all the resonance frequencies and the associated amplitudes.

To better visualize these results and to propose a more detailed analysis of the contribution of each order $\left[k_{1}, k_{2}\right]$, the responses of orders $\left[k_{1}, k_{2}\right]=[0,1]$ and $\left[k_{1}, k_{2}\right]=[1,0]$ are given in Figures $11(\mathrm{c}, \mathrm{d})$ and $11(\mathrm{e}, \mathrm{f})$, respectively. We notice a similar enlargement of the operating area for all the contributions of orders. This fact can be explained by the addition of uncertainty on the linear stiffness that induces a relatively similar effect to the responses of different orders $\left[k_{1}, k_{2}\right]=[1,0]$ and $\left[k_{1}, k_{2}\right]=[0,1]$, as indicated in Equation (35). As previously explained in section 3.3.3, the classical characteristic of a nonlinear system with contact is observed: a break of slope of the first and second resonance peaks (around $[11.8 ; 12.4] \mathrm{Hz}$ and $[16.4 ; 17.7] \mathrm{Hz}$ ) is observed due to the transition between the non contact state and contact state. The same phenomena is also visible for the third peak resonances at $32 \mathrm{~Hz}$ with a less marked feature. For these three resonance peaks, uncertainty in the linear stiffness of the two degree-of-freedom induces a variation of the resonance frequency and a shift of the three resonance peaks to the right. Here again, using the Stochastic-MHBM, it is possible to describe the three possible nonlinear responses due to the hardening effect, with low or high amplitudes in the interval between $[12.4 ; 13.1] \mathrm{Hz}$ and $[17.7 ; 18.7] \mathrm{Hz}$ for a given frequency.

The comparison of the random nonlinear response from the Stochastic-MHBM (with AFTPC) and the reference one evaluated by the simulations of Monte-Carlo and the Multi-dimensional Harmonic Balance method, validates the Stochastic-MHBM methodology for a mechanical system with non-regular nonlinearity and subjected to two incommensurable multi-excitations.

\subsubsection{Case 7: uncertainty in the friction element with incommensurable multi-excitations}

For case 7, the nonlinear term is chosen to be the frictional interface (as previously indicated in section 3.3.4) and we investigate the effect of a variation on the friction coefficient $\delta_{\mu}=5 \%$ with the mean value of the friction coefficient $\bar{\mu}=0.4$. All the other physical parameters are kept deterministic and the multiple excitation of incommensurable frequencies $\omega_{1}$ et $\omega_{2}$ (with $\frac{\omega_{2}}{\omega_{1}}=\frac{1}{\sqrt{2}}$ ) is applied on the system.

Figure 12(a,c,e) show the results for the nonlinear quasi-periodic responses and the responses of orders $\left[k_{1}, k_{2}\right]=[0,1]$ and $\left[k_{1}, k_{2}\right]=[1,0]$ by applying the Stochastic-MHBM (with AFTPC). Zooms are plotted in Figures 12(b,d,f) with adding the Monte Carlo simulations. As previously explained in section 3.3.2, only some samples are plotted that shows in Figures 12(b,d,f) the correlation between the MCS and Stochastic-HBM results. Due to the frictional nonlinear element, a softening effect with a small shift of the first and second resonance peaks (at $12 \mathrm{~Hz}$ and $17 \mathrm{~Hz}$ ) is detected. Once again, this observation is consistent with the trends already known on the dissipative effects due to friction. As previously observed in section 3.3.4, uncertainty on the coefficient of friction induces small variation for both the response amplitudes of all harmonic components and the associated frequencies.

The remarks previously stated on the efficiency and robustness of the proposed Stochastic-MHBM remain still valid in the case 
of the two degree-of-freedom with uncertainty in the nonlinear frictional interface and subjected to multiple incommensurable frequencies: this allows us to conclude on the robustness of the Stochastic-MHBM with AFTPC to estimate the random quasiperiodic response and overall contributions of harmonics.

\section{Conclusion}

In this paper, a study devoted to calculating the random nonlinear dynamical response of a two degree-of-freedom system with regular and non-regular nonlinearities is presented. Due to the fact that the use of classical methods for nonlinear systems with uncertainties can be rather expensive and requires considerable resources both in terms of computation time and data storage, a new concept called the Stochastic Multi-dimensional Harmonic Balance (Stochastic-MHBM) is proposed and discussed. The main originality and objective of this technique are to extract the quasi-periodic stochastic behavior of mechanical systems by using approximations. The principal idea is to replace the stochastic nonlinear responses and the stochastic nonlinear forces in the dynamical systems by constructing linear functions such as Fourier series on the chaos basis. The comparison of the results from this stochastic nonlinear approach and the reference one, combining the simulations of Monte Carlo and the Multi-dimensional Harmonic Balance method, for different cases of uncertainties, validates the proposed methodology. In particular, it was shown that the process is able to capture all the possible response levels when the response of the nonlinear system is not unique.

In conclusion, the proposed method offers a powerful technique for nonlinear systems with uncertainties and various nonlinearities. Future work to be considered consists of applying this new stochastic nonlinear method for complex systems and practical cases in the field of mechanical engineering.

\section{Acknowledgments}

The first author gratefully acknowledges the French Education Ministry which supports this research. Jean-Jacques Sinou gratefully acknowledges the financial support of the French National Research Agency through the Young Researcher program ANR07-JCJC-0059-01-CSD 2.

\section{References}

[1] J.A.S. Witteveen, A. Loeven, S. Sarkar, and H. Bijl. Probabilistic collocation for period-1 limit cycle oscillations. Journal of Sound and Vibration, 311(1-2):421-439, March 2008.

[2] A. Nayfeh. Perturbation Methods. John Wiley and Sons, London, 1973.

[3] H. Benaroya and M. Rehak. Finite element methods in probabilistic structural analysis: A selective review. Applied Mechanics Reviews, 41(5):201-213, 1988.

[4] F. Yamazaki, M. Shinozuka, and G. Dasgupta. Neumann expansion for stochastic finite element analysis. Journal of Engineering Mechanics, ASCE, 114(8):1335-1354, 1988.

[5] R. Ghanem and P. Spanos. Stochastic Finite Elements: A Spectral Approach. Springer-Verlag, 1991.

[6] J. Didier, J-J. Sinou, and B. Faverjon. Study of the non-linear dynamic response of a rotor system with faults and uncertainties. Journal of Sound and Vibration, 331:671-703, 2012.

[7] J. Didier, J-J. Sinou, and B. Faverjon. Multi-dimensional harmonic balance with uncertainties applied to rotor dynamics. Journal of Vibration and Acoustic, 134(6):061003, 17 pages, 2012.

[8] E. Sarrouy and J-J. Sinou. Non-linear periodic and quasi-periodic vibrations in mechanical systems - On the use of the harmonic balance methods, Advances in Vibration Analysis Research. Farzad Ebrahimi (Ed.), INTECH Publisher, 2011.

[9] K. Worden, G. Manson, T.M. Lord, and M.I. Friswell. Some observations on uncertainty propagation through a simple nonlinear system. Journal of Sound and Vibration, 288(3):601-621, December 2005.

[10] N. Coudeyras, S. Nacivet, and J-J. Sinou. Periodic and quasi-periodic solutions for multi-instabilities involved in brake squeal. Journal of Sound and Vibration, 328(4-5):520-540, December 2009.

[11] Y-B. Kim and Choi S-K. A multiple harmonic balance method for the internal resonant vibration of a non-linear jeffcott rotor. Journal of Sound and Vibration, 208(3):745-761, 1997.

[12] M. Guskov, J-J. Sinou, and Thouverez F. Multi-dimensional harmonic balance applied to rotor dynamics. Mechanics Research Communications, 35:537-545, 2008. 
[13] T. M. Cameron and J. H. Griffin. An alternating Frequency/Time domain method for calculating the Steady-State response of nonlinear dynamic systems. Journal of Applied Mechanics, 56(1):149-154, March 1989.

[14] J-J. Sinou. Non-linear dynamics and contacts of an unbalanced flexible rotor supported on ball bearings. Mechanism and Machine Theory, 44:1713-1732, 2009.

[15] M. Loève. Probability Theory, $4^{\text {th }}$ edition. Springer-Verlag, 1977.

[16] N. Wiener. The homogeneous chaos. American Journal of Mathematics, 60(4):897-936, October 1938.

[17] J-J. Sinou. Detection of cracks in rotor based on the $2 \times$ and $3 \times$ super-harmonic frequency components and the crackunbalance interactions. Communications in Nonlinear Science and Numerical Simulation, 13(9):2024-2040, November 2008.

[18] D. Laxalde, F. Thouverez, J-J. Sinou, and J-P. Lombard. Qualitative analysis of forced response of blisks with friction ring dampers. European Journal of Mechanics - A/Solids, 26(4):676-687, July 2007.

[19] B. Al Sayed, E. Chatelet, S. Baguet, and G. Jacquet-Richardet. Dissipated energy and boundary condition effects associated to dry friction on the dynamics of vibrating structures. Mechanism and Machine Theory, 46(4):479-491, April 2011. 


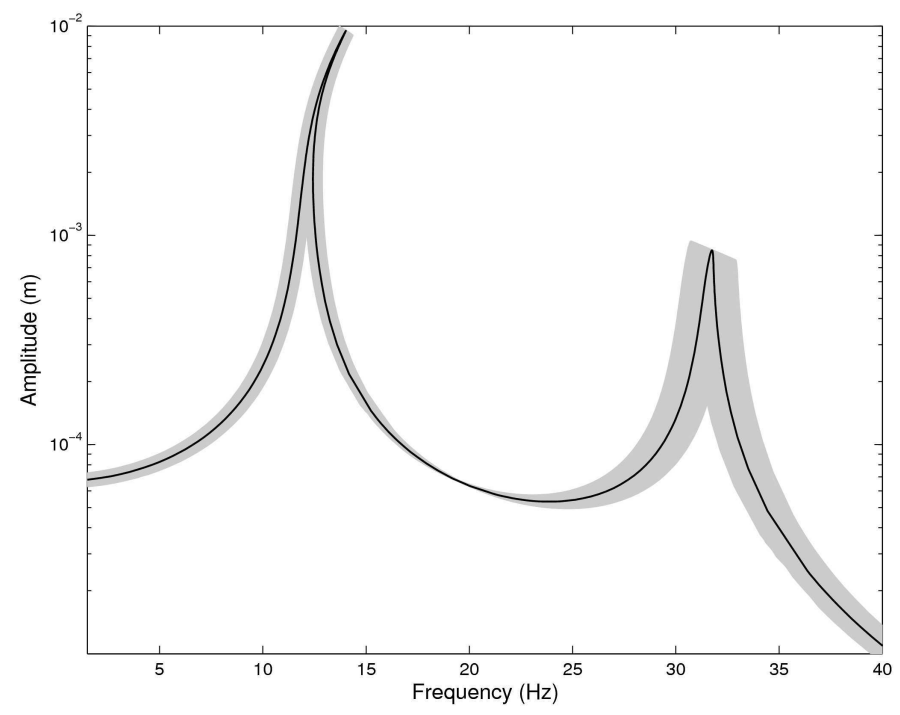

(a)

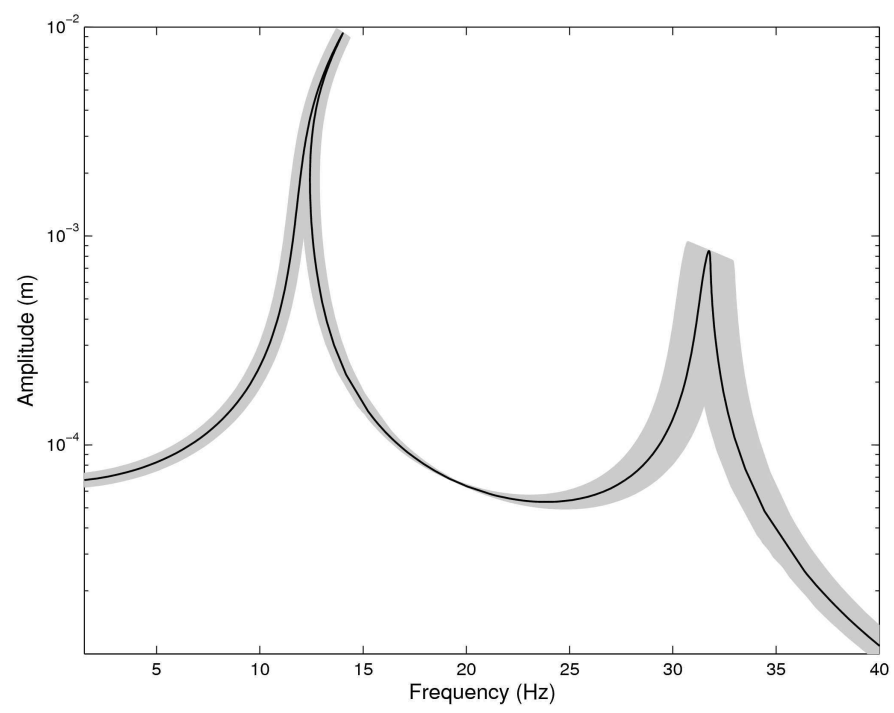

(c)

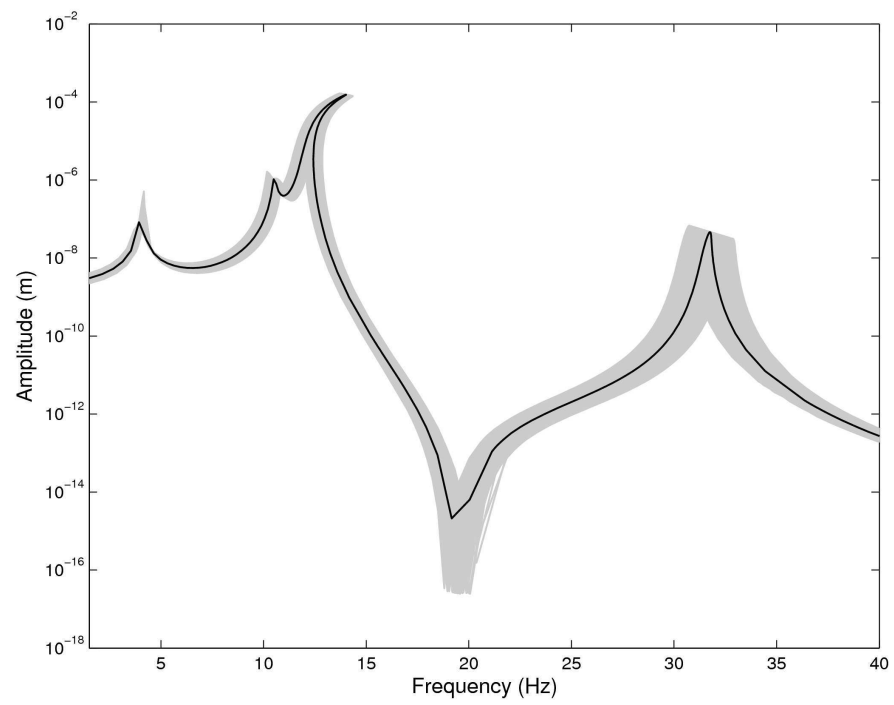

(e)

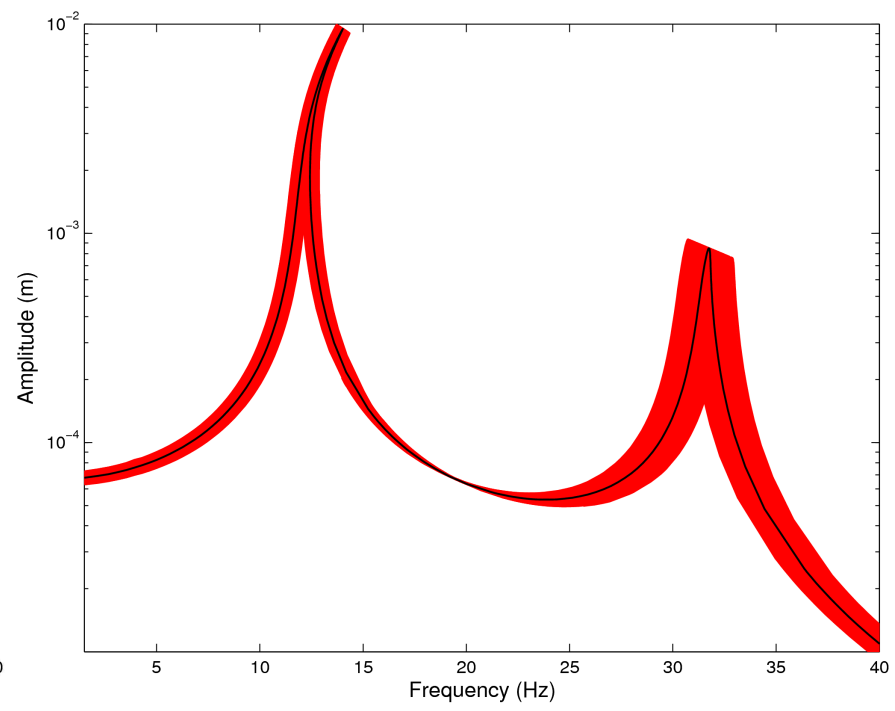

(b)

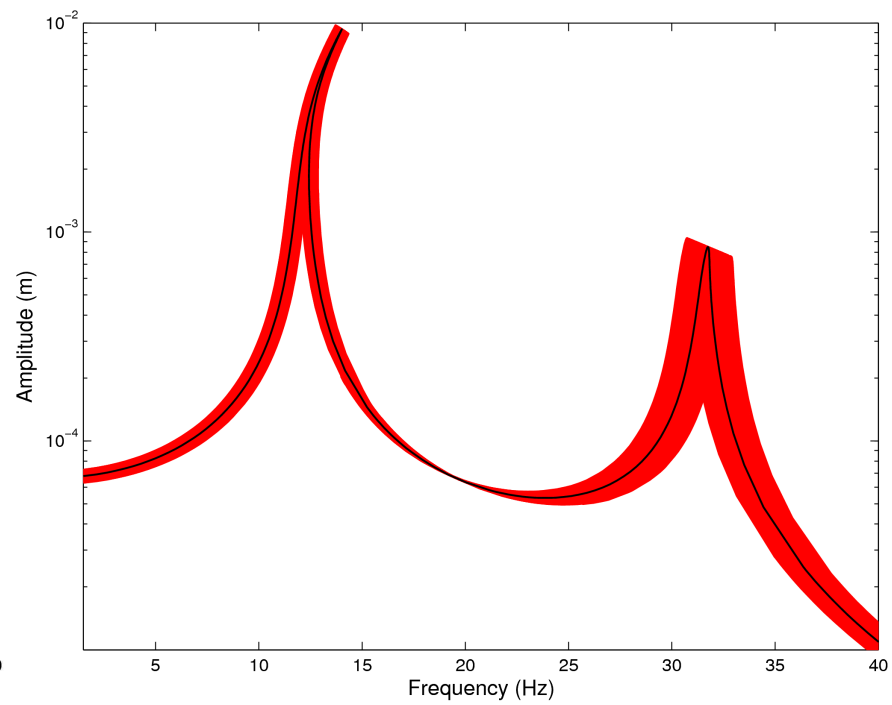

(d)

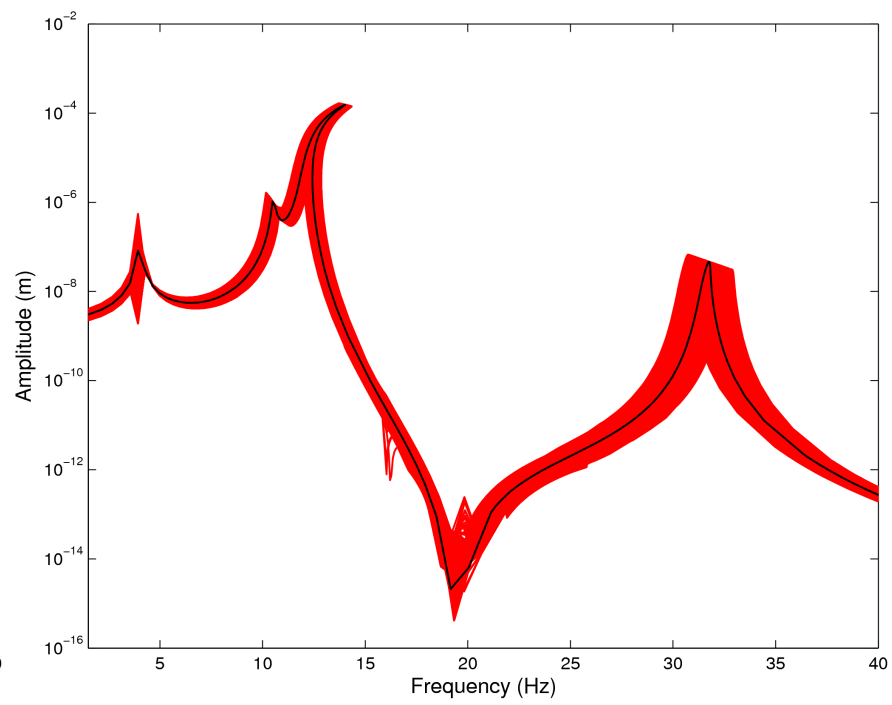

(f)

Fig. 5: Frequency Response Functions of the random nonlinear response for case 1: (a,c,e) MCS (plots in grey); (b,d,f) StochasticHBM (plots in red); deterministic response (black); (a,b) global nonlinear response; (c,d) $1 \times$ harmonic component ; (e,f) $3 \times$ harmonic component. 


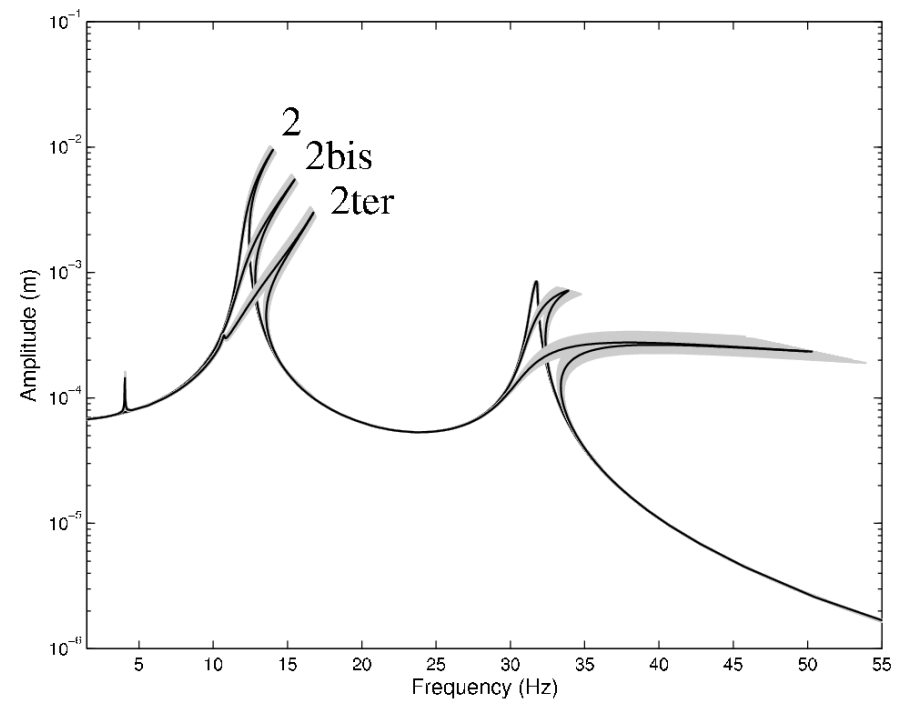

(a)

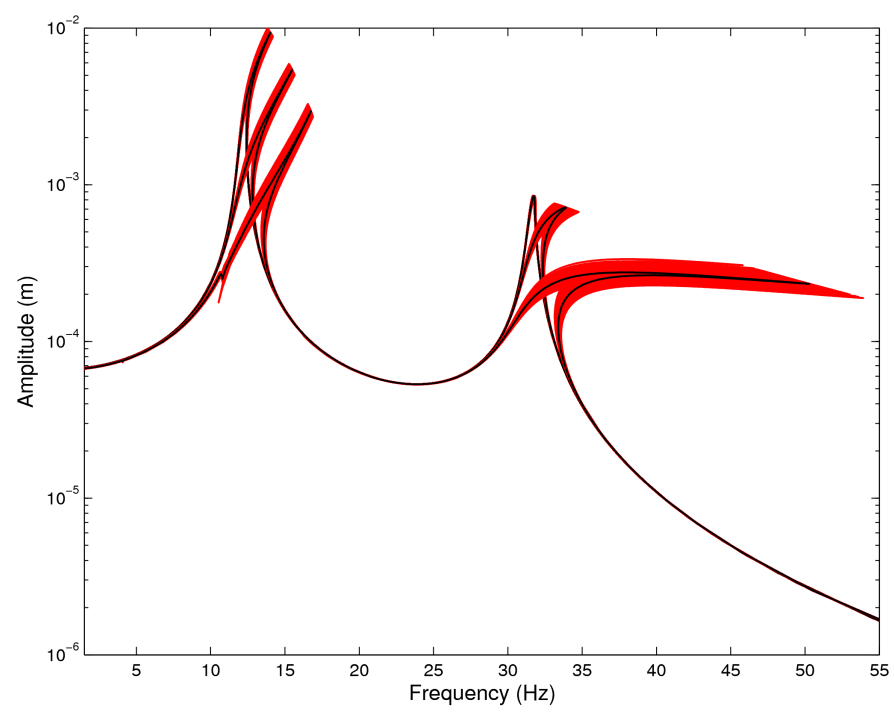

(c)

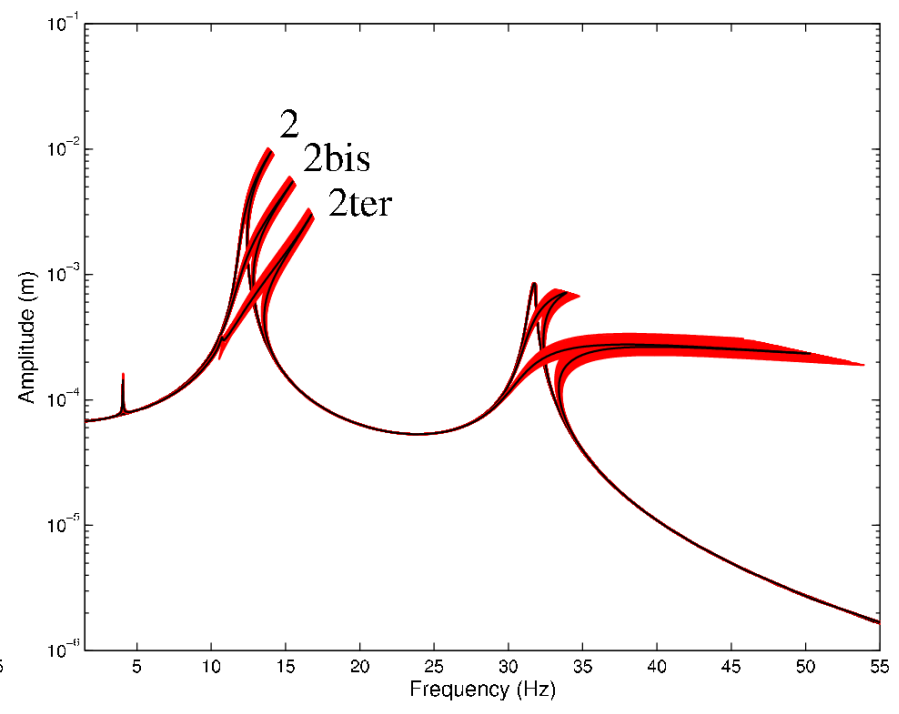

(b)

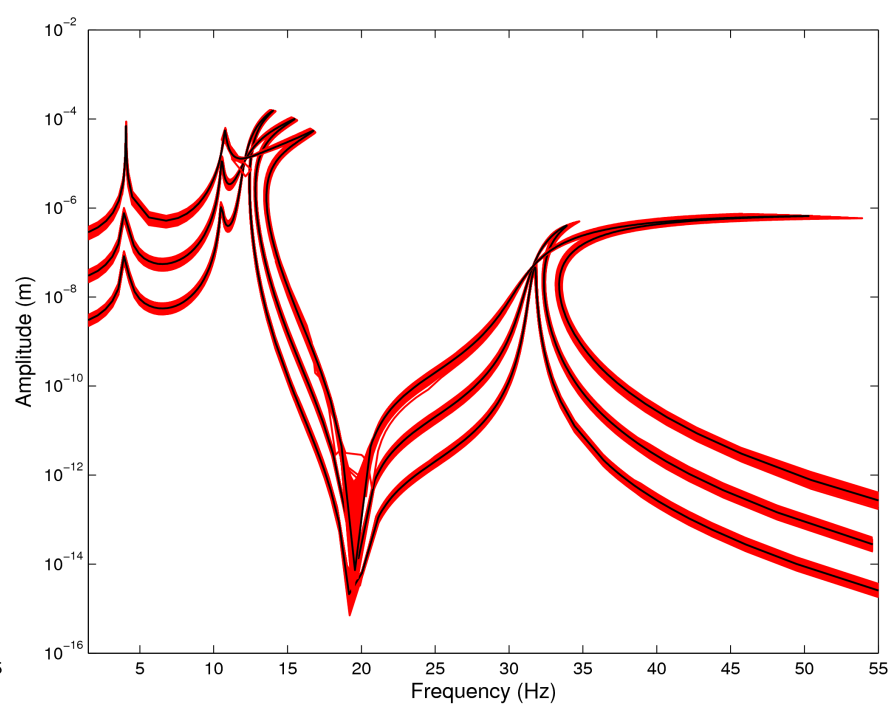

(d)

Fig. 6: Frequency Response Functions of the random nonlinear response for cases 2, 2bis and 2ter: (a) MCS (plots in grey); (b,c,d) Stochastic-HBM (plots in red); deterministic response (black); (a,b) global nonlinear response; (c) $1 \times$ harmonic component ; (d) $3 \times$ harmonic component. 


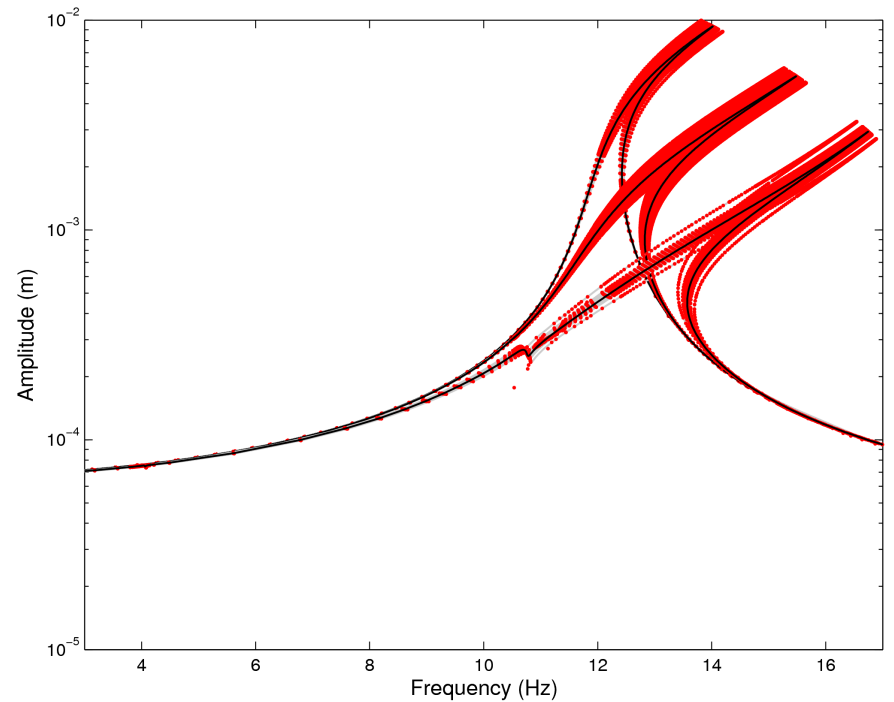

(a)

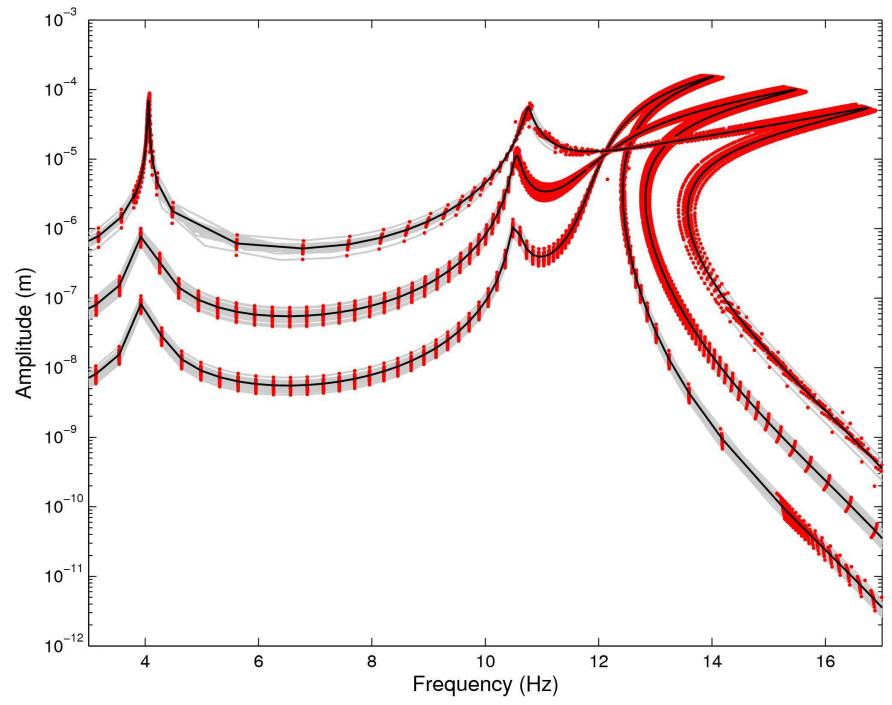

(b)

Fig. 7: Zoom of the Frequency Response Functions of the random nonlinear response for cases 2, 2bis and 2ter: MCS (plots in grey); Stochastic-HBM (plots in red); deterministic response (black); (a) $1 \times$ harmonic component; (b) $3 \times$ harmonic component.

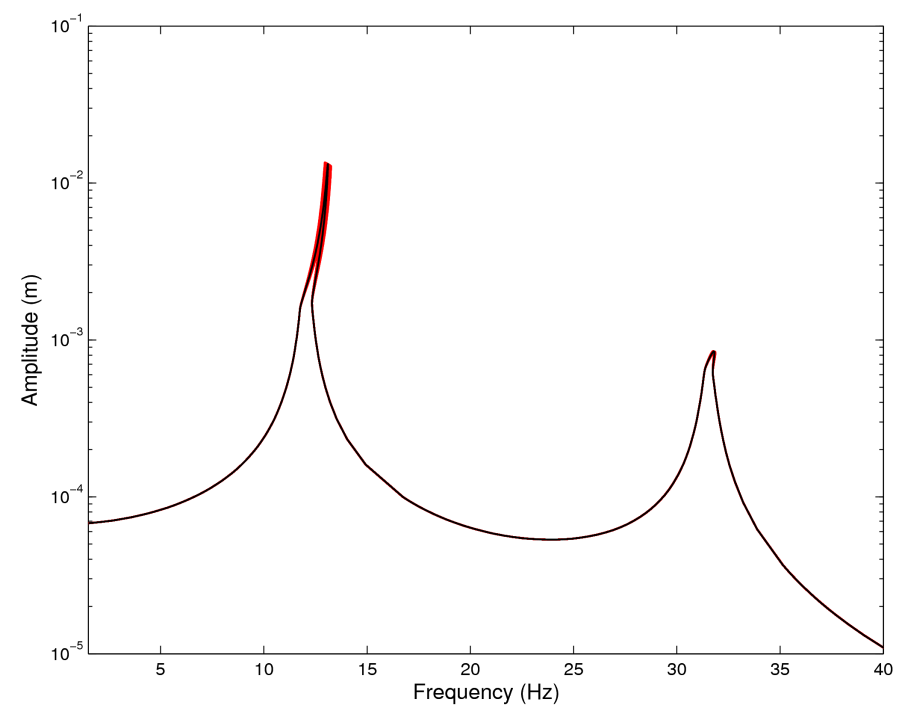

(a)
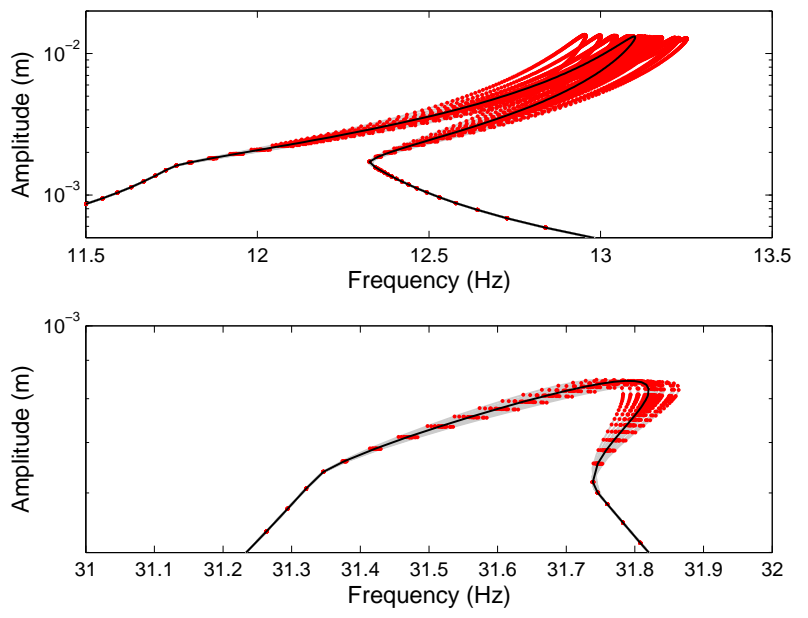

(b)

Fig. 8: Frequency Response Functions of the random nonlinear response for case 3: (a) Stochastic-HBM (plots in red); (b) Zooms : deterministic response (black), MCS (grey), Stochastic-HBM (red). 


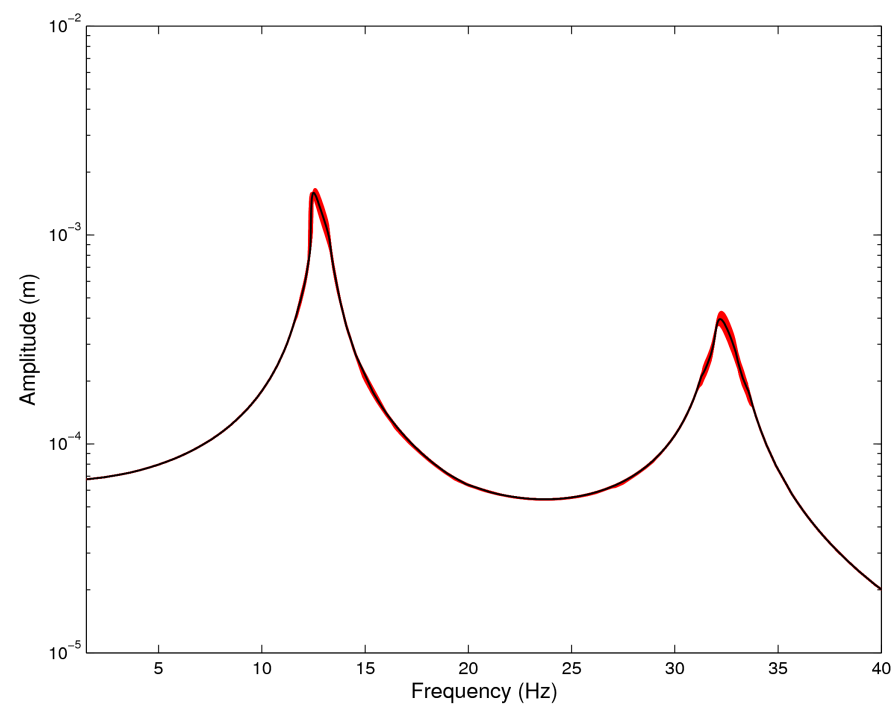

(a)
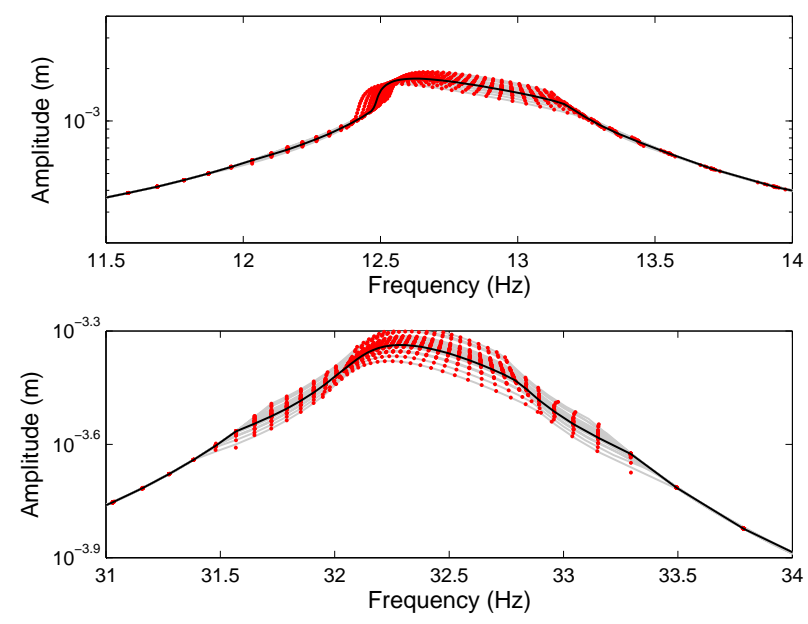

(c)
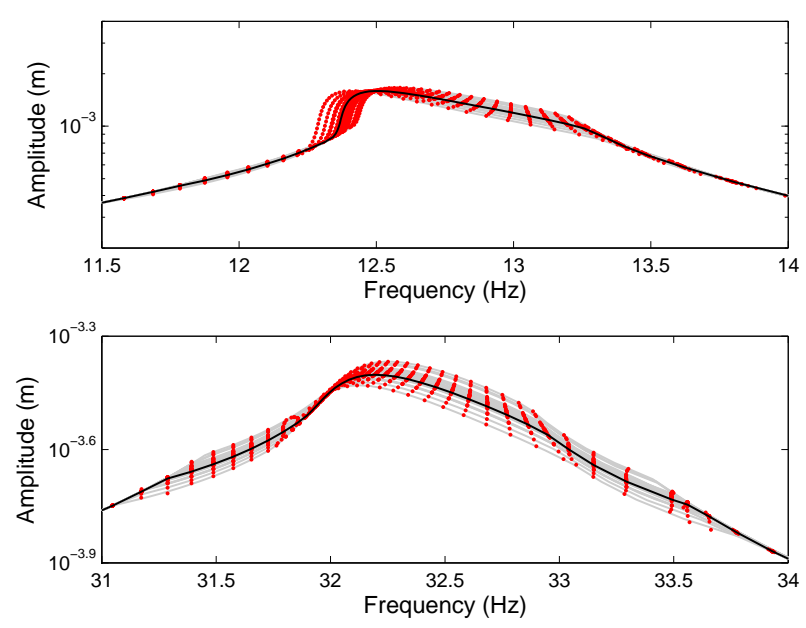

(b)
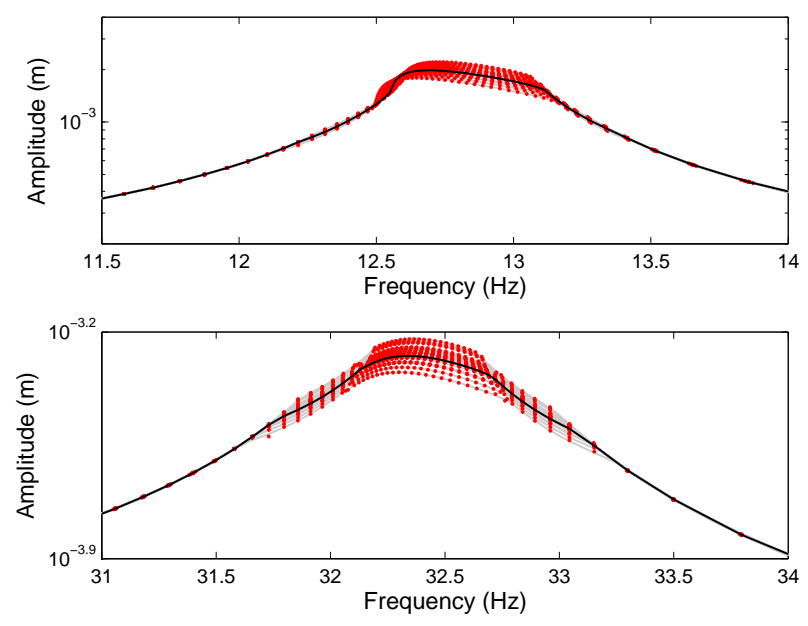

(d)

Fig. 9: Frequency Response Functions of the random nonlinear response for cases 4, 4bis and 4ter: Stochastic-HBM (plots in red); MCS (grey); deterministic response (black); (a) case 4; (b) Zooms of the FRF for case 4; (c) Zooms of the FRF for case 4bis; (d) Zooms of the FRF for case 4ter. 


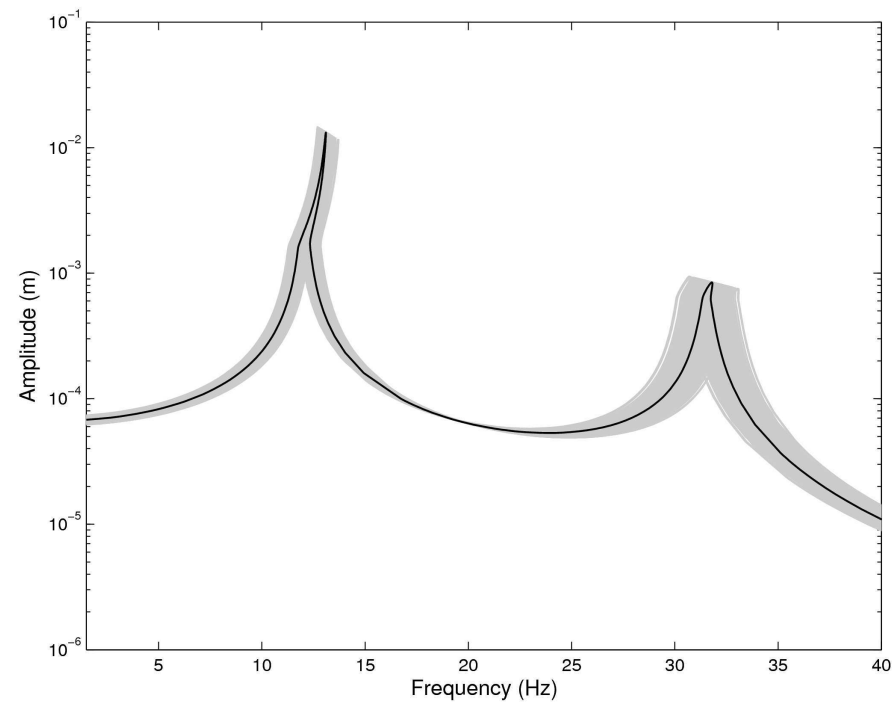

(a)

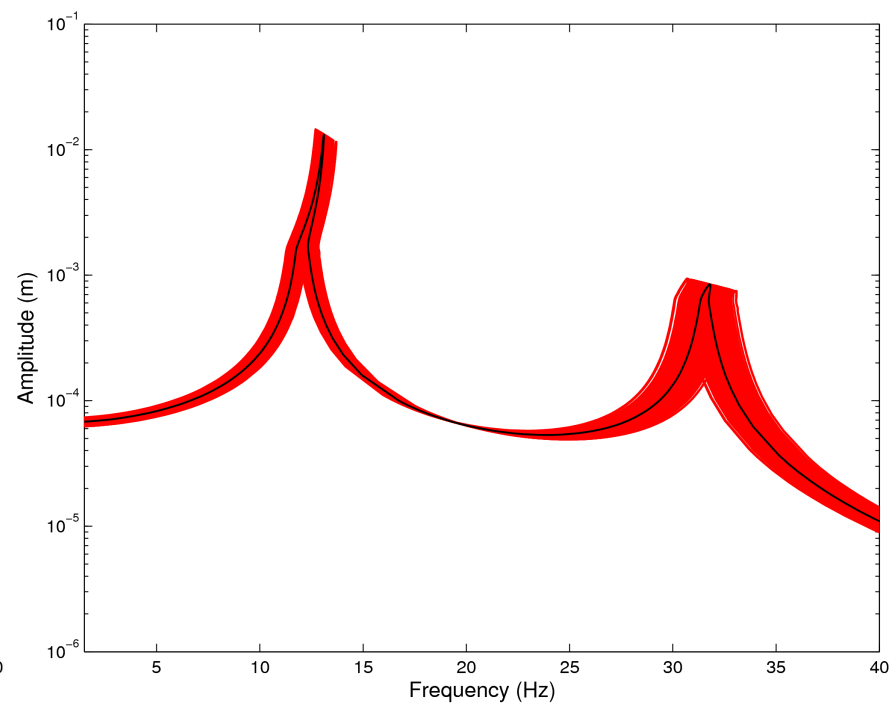

(b)

Fig. 10: Frequency Response Functions of the random nonlinear response for case 5: (a) MCS (plots in grey); (b) StochasticHBM (plots in red); deterministic response (black). 


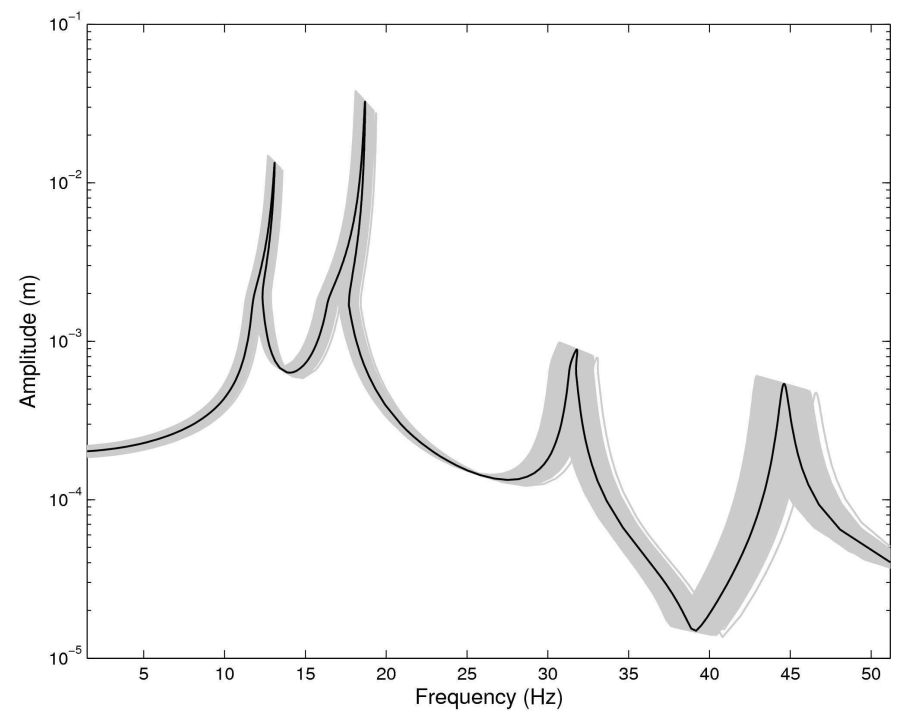

(a)

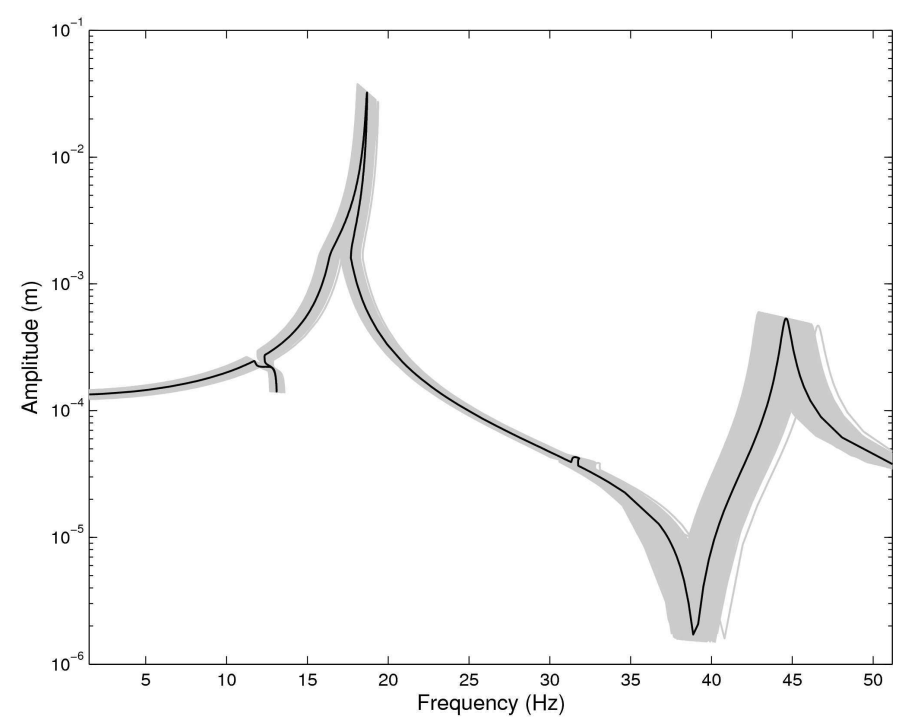

(c)

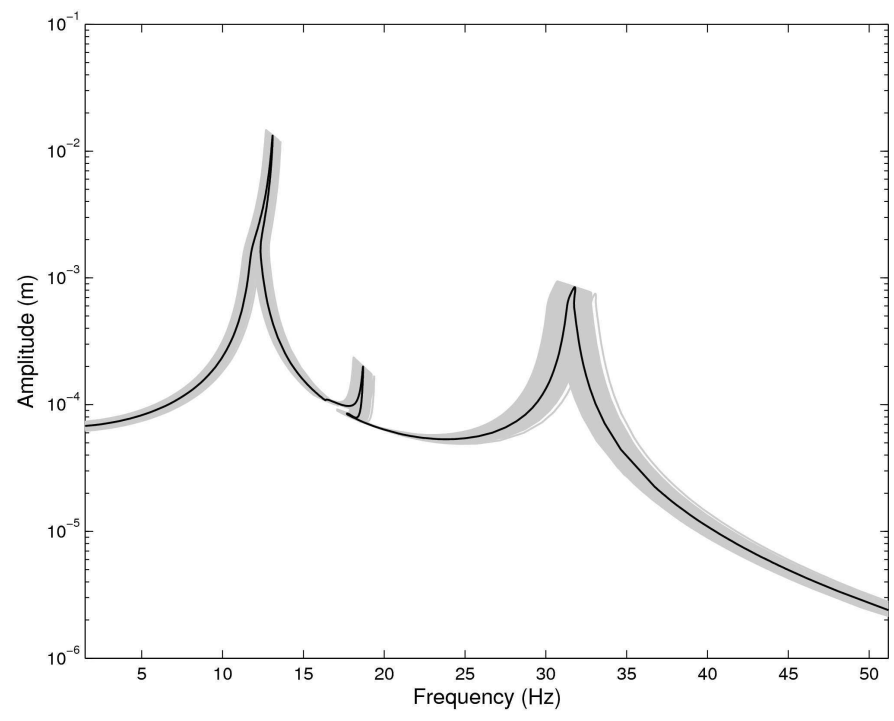

(e)

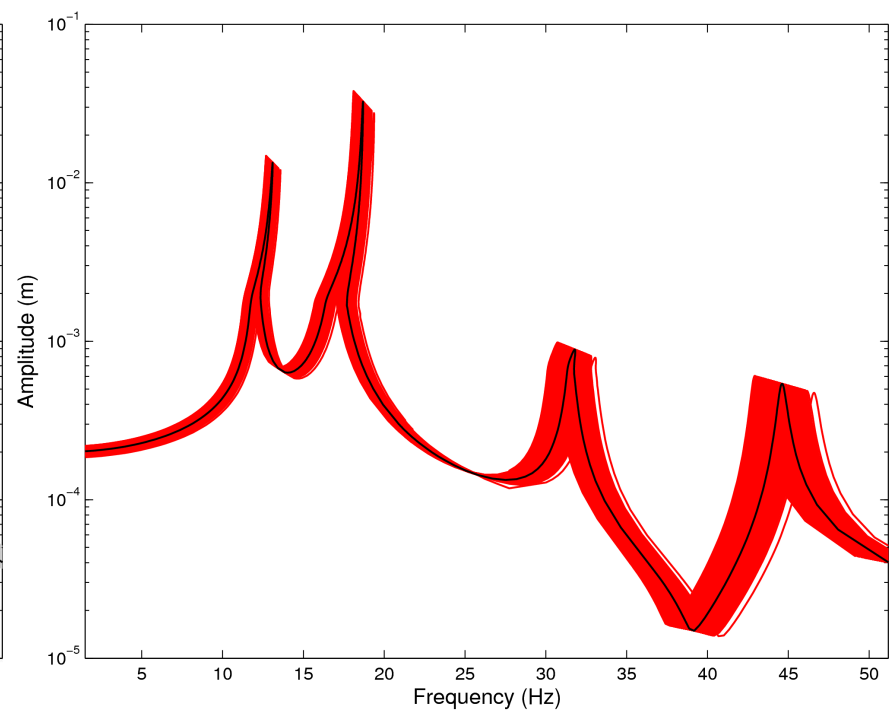

(b)

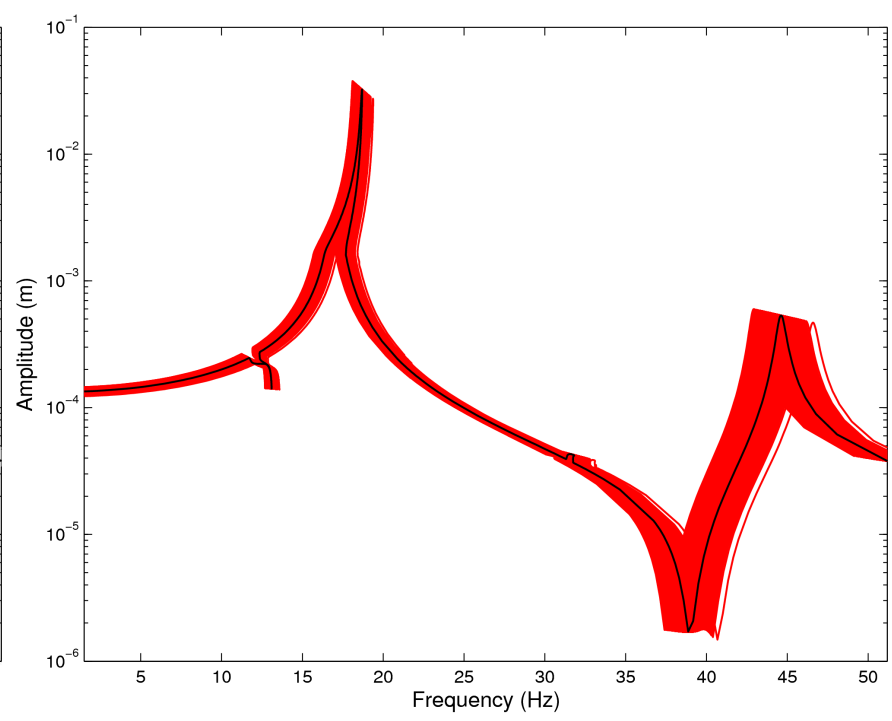

(d)

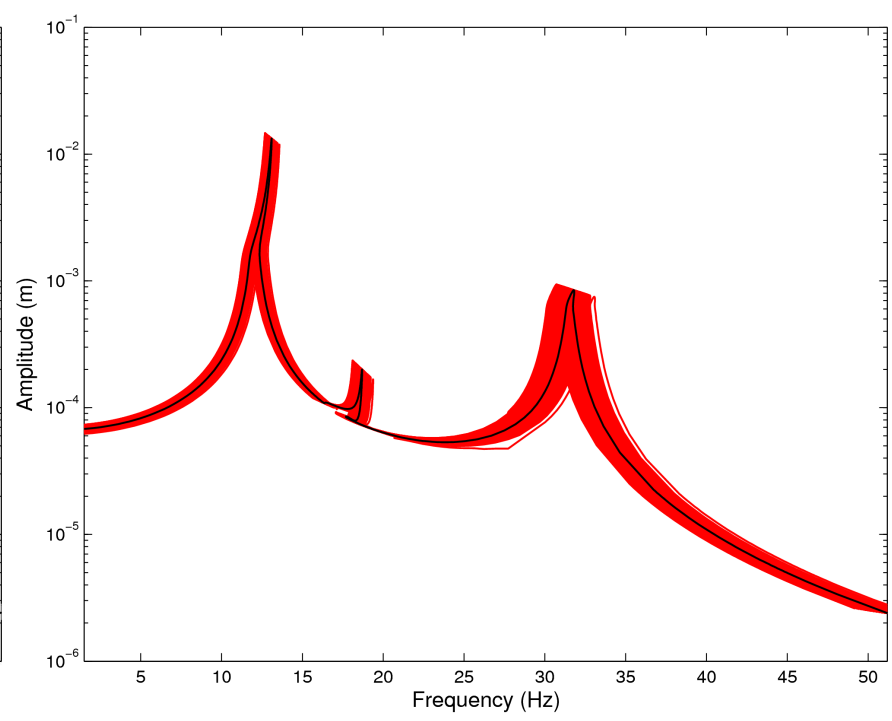

(f)

Fig. 11: Frequency Response Functions of the random nonlinear response for cases 6: (a,c,e) MCS (plots in grey); (b,d,f) Stochastic-HBM (plots in red); deterministic response (black); (a,b) global nonlinear response; (c,d) [0,1] harmonic component; (e,f) $[1,0]$ harmonic component. 


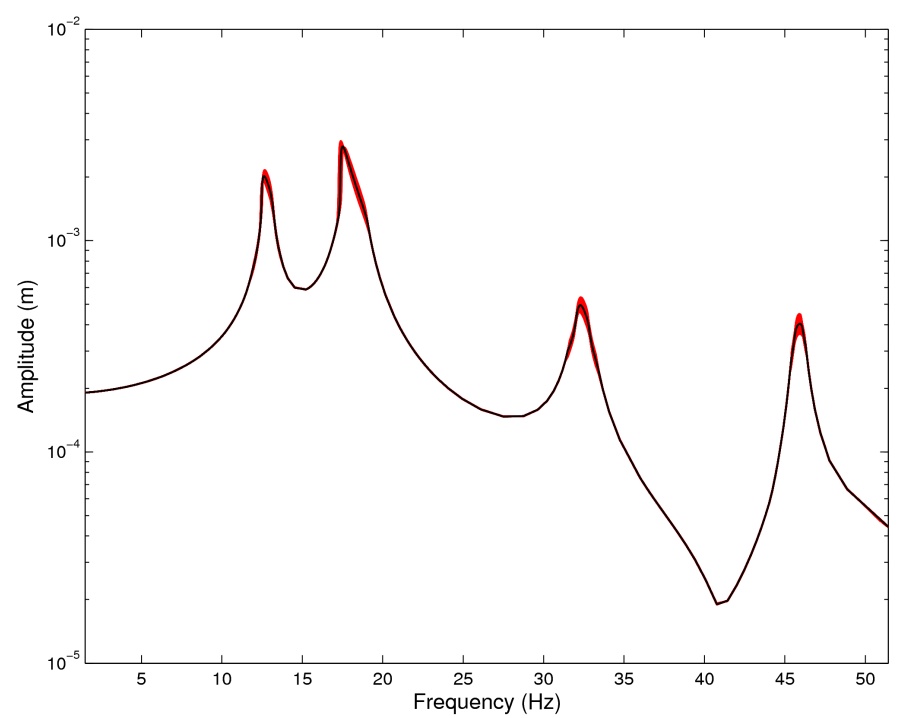

(a)

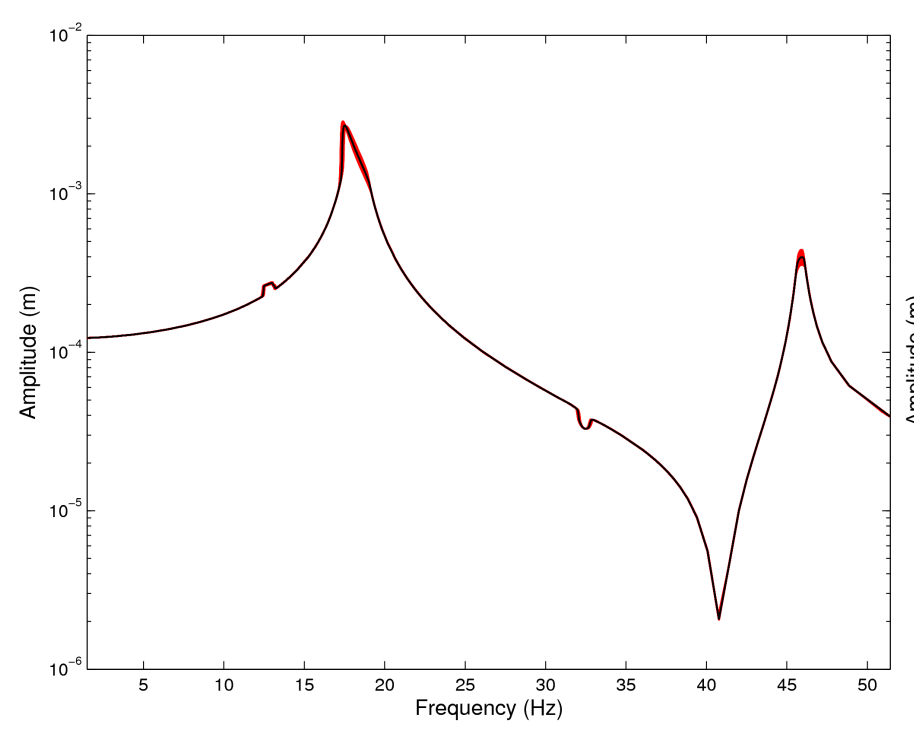

(c)

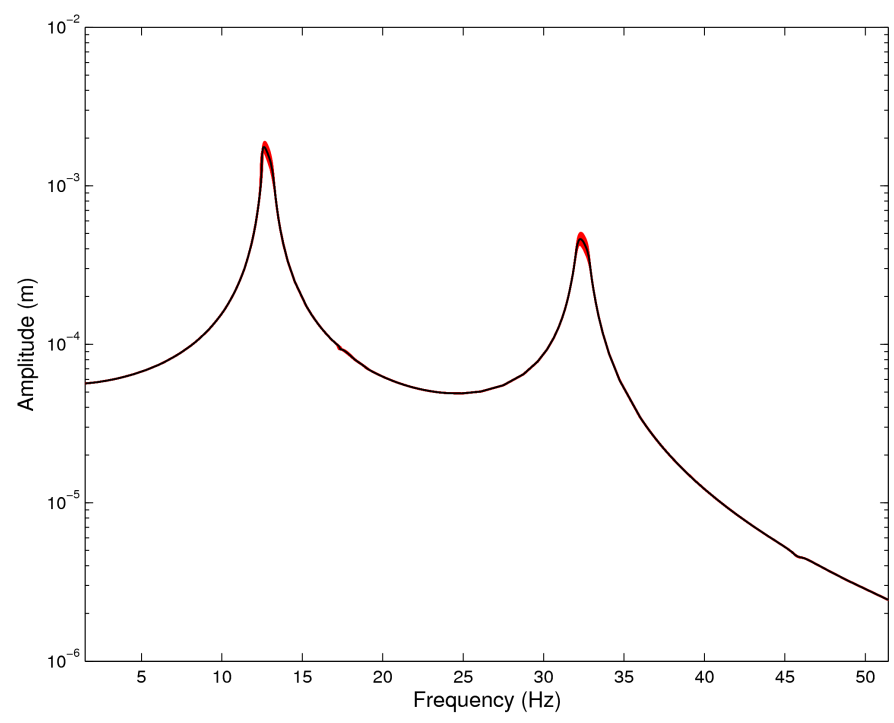

(e)

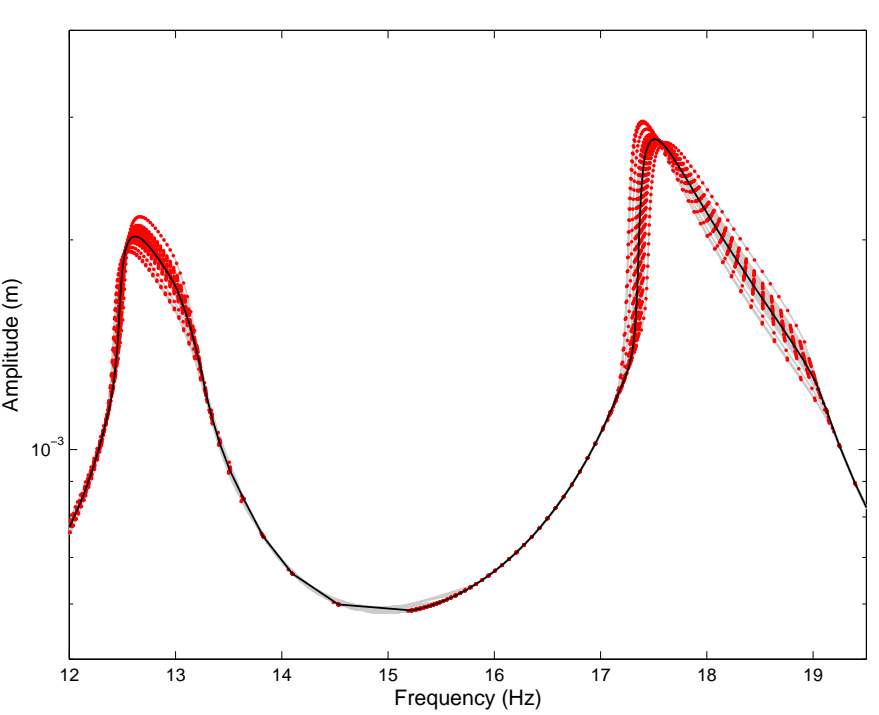

(b)

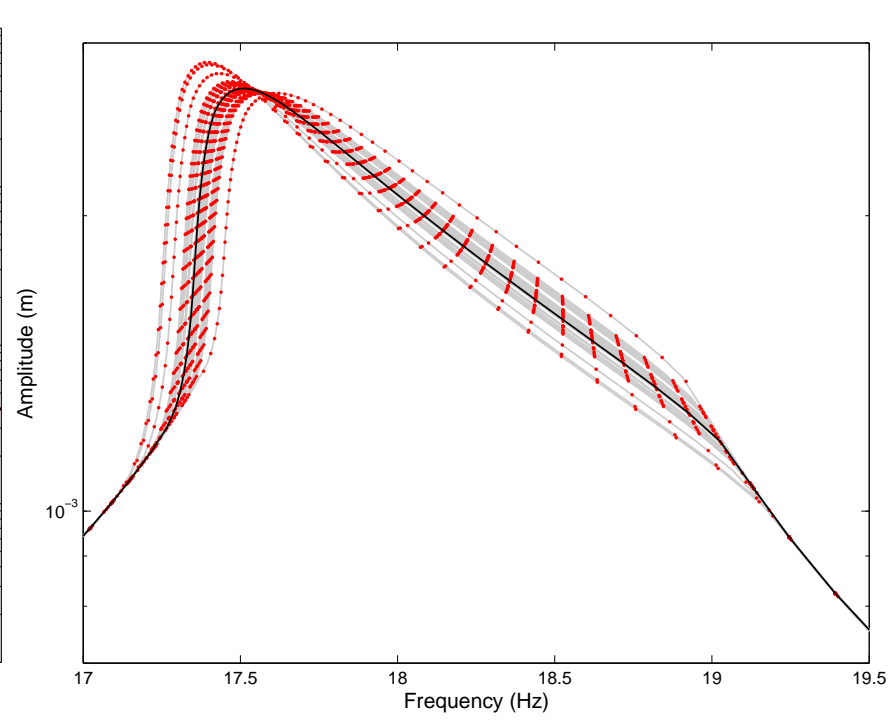

(d)

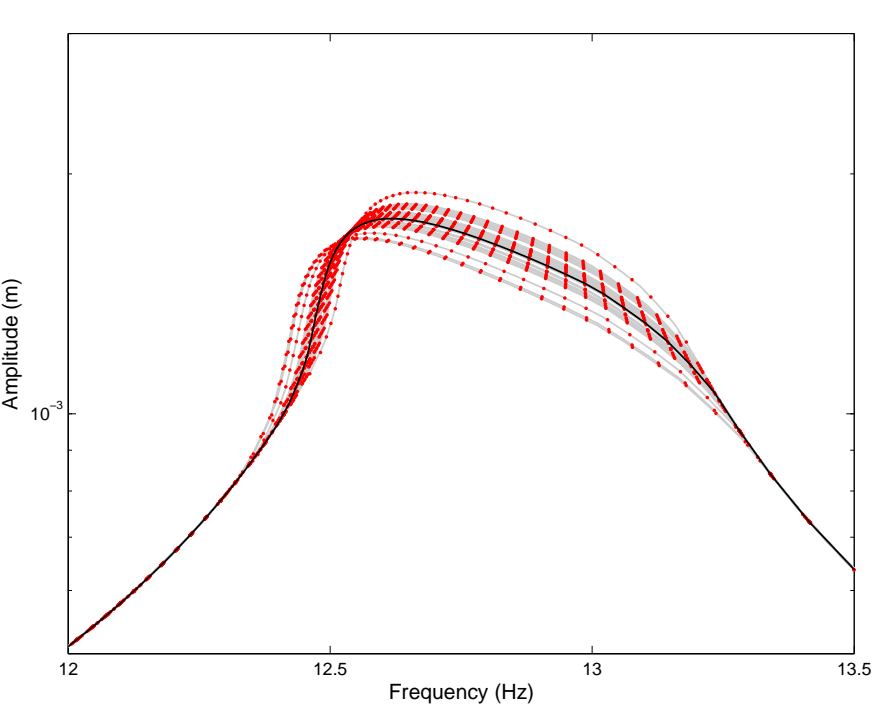

(f)

Fig. 12: Frequency Response Functions of the random nonlinear response for case 7: (a,c,e) Stochastic-HBM (plots in red); (b,d,f) Zooms with MCS (grey), Stochastic-MHBM (red) and deterministic response (black); (a,b) global nonlinear response; (c,d) $[0,1]$ harmonic component; $(\mathrm{e}, \mathrm{f})[1,0]$ harmonic component. 